\title{
Universal-dephasing-noise injection via Schrödinger-wave autoregressive moving-average models
}

\author{
Andrew Murphy, ${ }^{1}$ Jacob Epstein ${ }^{\oplus},{ }^{1}$ Gregory Quiroz, ${ }^{1}$ Kevin Schultz, ${ }^{1}$ Lina Tewala $\odot,{ }^{1,2}$ Kyle McElroy, ${ }^{1}$ Colin Trout,${ }^{1}$ \\ Brian Tien-Street $\odot,{ }^{1}$ Joan A. Hoffmann, ${ }^{1}$ B. D. Clader, ${ }^{1}$ Junling Long, ${ }^{3}$ David P. Pappas, ${ }^{3}$ and Timothy M. Sweeney ${ }^{1}$ \\ ${ }^{1}$ Johns Hopkins University Applied Physics Laboratory, Laurel, Maryland 20723, USA \\ ${ }^{2}$ Thomas C. Jenkins Department of Biophysics, Johns Hopkins University, Baltimore, Maryland 21218, USA \\ ${ }^{3}$ National Institute of Standards and Technology, Boulder, Colorado 80305, USA
}

(Received 6 July 2021; revised 2 December 2021; accepted 7 December 2021; published 2 February 2022)

\begin{abstract}
We present and validate a method for noise injection of arbitrary spectra in quantum circuits that can be applied to any system capable of executing arbitrary single qubit rotations, including cloud-based quantum processors. As the consequences of temporally correlated noise on the performance of quantum algorithms are not well understood, the capability to engineer and inject such noise in quantum systems is paramount. To date, noise injection capabilities have been limited and highly platform specific, requiring low-level access to control hardware. We experimentally validate our method by comparing to a direct hardware-based noise-injection scheme. Using a combination of quantum noise spectroscopy and classical signal analysis, we show that the two approaches agree. These results showcase a highly versatile method for noise injection that can be utilized by theoretical and experimental researchers to verify, evaluate, and improve quantum characterization protocols and quantum algorithms for sensing and computing.
\end{abstract}

DOI: 10.1103/PhysRevResearch.4.013081

\section{INTRODUCTION}

As qubit technology advances into the noisy intermediatescale quantum era, spurred by quantum algorithms that promise advantages over classical alternatives, quantum hardware is progressively able to support circuits that are increasingly complex in circuit depth and system size. While numerous physical technologies and architectures are being developed, each system suffers from limitations and sensitivities due to environmental and systematic noise sources. Ultimately, these noise sources lead to computational errors that destroy the efficacy of a quantum computation. For this reason, a significant amount of research has focused on characterizing [1-8] and addressing (i.e., avoiding [9], suppressing [10-13], or correcting [14-18]) noise in quantum systems. These efforts have been complemented by evaluations of quantum algorithmic resilience to specific noise sources [19-23].

Engineered noise provides a necessary capability for investigating the effects of noise in a controlled environment. Beyond providing a means for validation and verification of system characterization protocols, engineered noise allows one to examine the susceptibility and robustness of noise characterization and mitigation protocols to particular noise types [24]. Furthermore, when sufficiently versatile, engineered noise can provide key insights into inherent noise resilience and vulnerabilities of quantum algorithms to targeted noise

Published by the American Physical Society under the terms of the Creative Commons Attribution 4.0 International license. Further distribution of this work must maintain attribution to the author(s) and the published article's title, journal citation, and DOI. sources. In these ways, engineered noise enables the development of robust system characterization techniques, noise mitigation protocols, and error-resilient quantum algorithms, all of which can be considered necessary for achieving faulttolerant quantum computation. To date, engineered noise has been achieved in a few experimental platforms but a standard method for generating well-defined noise of arbitrary spectra has yet to be identified. Furthermore, methods for injecting engineered noise have been highly platform specific [4,5], with particular focus on laboratory-based experimental systems of superconducting transmon qubits [4,5] or trapped ions [25]. These noise-injection protocols require either a specific device design [4,5] or control of specific in-house hardware platforms [25].

In this paper, we present a platform-agnostic technique for injecting engineered dephasing noise of arbitrary spectra based on the Schrödinger wave autoregressive moving average (SchWARMA) model [26]. A generalization of the autoregressive moving average (ARMA) models used in classical signal processing, SchWARMA models have been previously used to numerically simulate spatiotemporally correlated noise processes in multiqubit systems. Exploiting the framework of SchWARMA-based numerical simulation, we show that the concept of inserting SchWARMA-designed error gates into an ideal quantum circuit can be leveraged for noise injection. This is a key advantage of SchWARMA over other noise-injection techniques, making it implementable in any situation where access to arbitrary single-qubit rotation gates is provided, including cloud devices such as the IBM Quantum Experience (IBMQE) [27]. Focusing, in particular, on phase noise, we show that SchWARMA can be utilized to introduce temporally correlated dephasing errors in real physical systems and that the SchWARMA approach has excellent predictive power as well. While this paper centers 
(a)

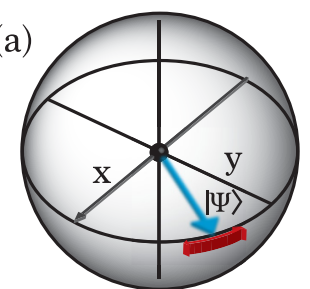

Noisy Qubit

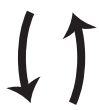

Noisy Master Clock

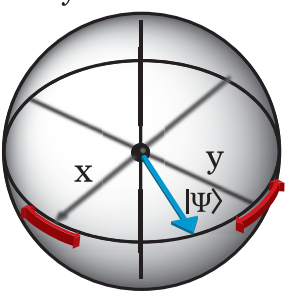

(b)
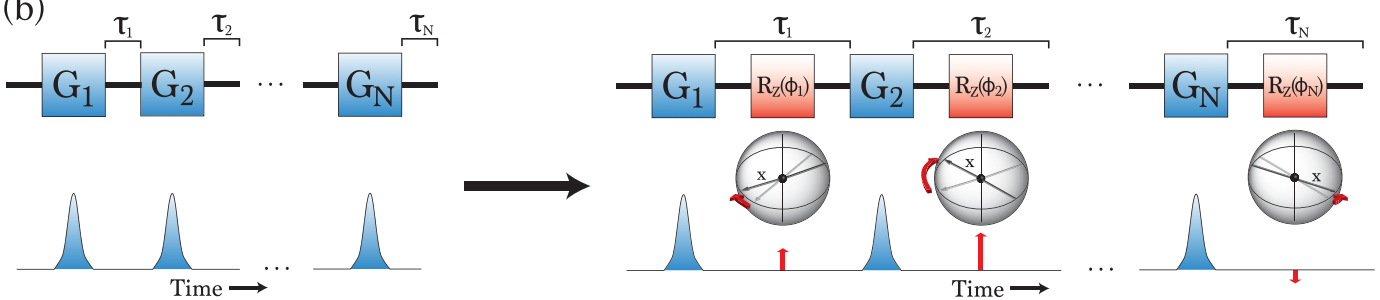

(c)

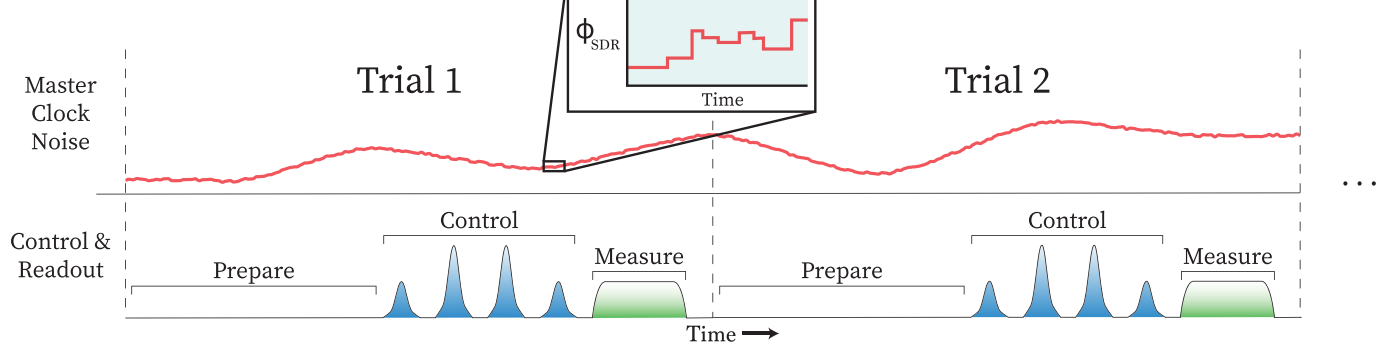

FIG. 1. Implementation of SchWARMA. (a) Phase noise on a qubit under ideal measurement conditions (upper Bloch sphere) is simulated by imparting controlled errors on the experimental clock (i.e., the reference frame) with no intentional additional noise on the qubit (lower Bloch sphere). (b) Gate injection. SchWARMA models an ideal quantum circuit in a noisy environment by interleaving error gates between each control pulse. We implement these error gates as instantaneous rotations of the qubit's reference frame. (c) SDR-based noise injection. In the SDR setup, the SDR signal is mixed with control pulses to impart errors in the control signal. The phase of the SDR signal is updated discretely, every $100 \mathrm{~ns}$, but the signal itself runs continuously over all shots in an experiment. Unlike gate-injection experiments, each shot in an SDR-based noise injection experiment will have a unique phase trajectory of the control signal.

around stationary Gaussian phase noise, we emphasize that the SchWARMA approach is far-reaching and enables injection of control amplitude noise, as well as nonstationary, non-Gaussian, and spatiotemporally correlated noise.

To validate the SchWARMA model as both a statistical model for describing temporally correlated noise and as a generative model for mimicking phase noise on a qubit, we develop and implement two SchWARMA-motivated noise injection approaches: (1) a gate-based approach-faithful to the original SchWARMA concept-that inserts correlated phase errors into an ideal circuit and (2) a low-cost hardware implementation that uses a software defined radio (SDR) to impart errors directly on the master clock. Approach (2) is less universal and not platform agnostic, requiring direct access to hardware for implementation. However, unlike (1), method (2) allows for direct measurement of the injected noise spectrum. For this reason, we present method (1) as a proof-of-principle for arbitrary phase-noise spectra injection using SchWARMA and present method (2) as a validator for method (1). The IBMQE serves as a test bed for approach (1), where hardware access is purposefully restricted to preset gates to convey the universality of SchWARMA noise injection on systems with limited access. In addition, an in-house transmon-based superconducting qubit system, which we will refer to as the Applied Physics Laboratory (APL) system, is employed as an evaluation test bed that allows for both gate-based and direct access scenarios to be examined and compared.

Using the qubit as a probe, the injected noise spectra is reconstructed via quantum noise spectroscopy (QNS) [1-5,28]. We find strong agreement between the reconstructed and in- jected noise spectra for both gate- and SDR-based approaches. The SDR approach is further validated by direct measurement of its output with a signal analyzer, displaying agreement with the desired spectrum. Additionally, we show that forward simulations of the corresponding SchWARMA models have strong correlation with the experimental data, closing the loop on the validation of the SchWARMA approach for modeling temporally correlated dephasing noise.

\section{NOISE INJECTION PROTOCOL}

\section{A. Dephasing noise injection}

The dephasing between a qubit and a master clock can be engineered through either dynamic control of a qubit's phase relative to the master clock or through the dynamic control of the phase of the master clock relative to the qubit. The former case is represented by the upper panel of Fig. 1(a) where the reference frame defined by the local oscillator (LO) is stationary and the qubit state wanders about the $z$ axis. Conversely, in the latter case, represented by the lower panel of Fig. 1(a), the qubit state is stationary while the reference frame wanders [29]. We artificially induce correlated phase noise using this latter method through dynamical control over the master clock on a single qubit system. The result is an effective qubit response that mimics that of a qubit subject to a temporally correlated classical noise environment. The phase modulation of the master clock $\phi(t)=\phi_{\mathrm{ctrl}}(t)+\phi_{\text {noise }}(t)$ is composed of the typical (potentially faulty) control phase $\phi_{\text {ctrl }}(t)$, with an additional $\phi_{\text {noise }}(t)$ representing the desired injected noise process. Next, we discuss how the SchWARMA formalism can be used to construct $\phi_{\text {noise }}(t)$. 


\section{B. Noise injection via SchWARMA}

ARMA models are widely used in the field of time-series analysis to model time correlations in data. SchWARMA provides a natural path for generalization of these classic techniques from signal processing to quantum information. While SchWARMA has been previously utilized as an approach for numerically simulating classical temporally correlated noise in quantum circuits, its utility can be readily extended to noise injection and model prediction.

The gate-based noise-injection protocol follows a similar recipe to the numerical approach demonstrated in Ref. [26], where the noise is injected via error gates interleaved in a quantum circuit, as demonstrated in Fig. 1(b). In the case of single qubit dephasing, a circuit with noise injection can be expressed as

$$
U(\boldsymbol{\phi})=R_{z}\left(\phi_{N}\right) G_{N} R_{z}\left(\phi_{N-1}\right) G_{N-1} \ldots R_{z}\left(\phi_{1}\right) G_{1},
$$

where $G_{j}$ denotes a single qubit operation in the noiseless circuit and the error gate $R_{z}\left(\phi_{j}\right)$ represents a $z$ rotation with time-correlated $\phi_{j}$. Upon specifying properties of the noise process, e.g., the mean and power spectral density, a SchWARMA model is used to generate a trajectory of temporally correlated phases $\phi=\left\{\phi_{1}, \ldots, \phi_{N}\right\}$. Averaging an observable or fidelity metric over many realizations of $\phi$ results in the desired dephasing behavior.

In the SchWARMA approach, temporal correlations in the phases $\phi_{j}$ are accomplished by applying linear filtering of samples of white Gaussian noise or, equivalently, driving an ARMA model with white Gaussian noise. The ARMA model coefficients $\left\{a_{i}\right\}$ (the autoregressive coefficients) and $\left\{b_{j}\right\}$ (the moving average coefficients) define the output phase $\phi_{k}$ from the white Gaussian input $w_{k}$ by

$$
y_{k}=\underbrace{\sum_{i=1}^{p} a_{i} y_{k-i}}_{A R}+\underbrace{\sum_{j=0}^{q} b_{j} w_{k-j}}_{M A} .
$$

This filtering approach yields a power spectrum $S_{y}(\omega)$ of

$$
S_{y}(\omega)=\frac{\left|\sum_{k=0}^{q} b_{k} \exp (-i k \omega)\right|^{2}}{\left|1+\sum_{k=1}^{p} a_{k} \exp (-i k \omega)\right|^{2}},
$$

and it is known that ARMA models can approximate any discrete-time power spectrum to arbitrary accuracy [30]. Here, these correlated phases are then converted to correlated error operators via $R_{z}\left(\phi_{k}\right)=\exp \left(-i \phi_{k} \sigma_{z} / 2\right)$, although the SchWARMA approach supports a much wider class of correlated error models [26]. In general, there are many ways to define ARMA coefficients in a way that achieves a desired power spectrum and such a discussion is beyond the scope of this paper. Here, bandpass signals are MA models defined by length 128 Hamming windows of the desired bandwidth and cosine modulated (as needed) to the appropriate center frequency, and then scaled to achieve the overall desired power. The outputs of independently driven models are added to produce multiband spectra. ARMA coefficients for $1 / f^{\alpha}$ noise were defined using the approach in Ref. [31].

This form of SchWARMA-based noise injection is equivalent to control master clock phase noise injection. In superconducting qubits, an $R_{z}(\phi)$ gate is implemented via a virtual frame change that simply updates the control phase [Fig. 1(a)]. In this sense, $\boldsymbol{\phi}$ represents a discretized implementation of $\phi_{\text {noise }}(t)$. When the sampling time of $\phi_{\text {noise }}(t)$ is the fundamental gate time, $t_{G}, \boldsymbol{\phi}$, and $\phi_{\text {noise }}(t)$ are identical. Note that all gates $G_{j}$ do not need to be equivalent in duration but rather just integer multiples of $t_{G}$. For example, if gate $G_{j}$ requires timing $n t_{G}$, the composition of $n$ error gates are appended to the circuit following $G_{j}$. Note that this sampling time $t_{G}$ limits the maximum frequency of noise that can be injected to $t_{G}^{-1} / 2$.

Below, we also introduce an SDR-based SchWARMA noise injection technique that is distinct from the gate-based approach in that it occurs simultaneously and asynchronously with the control. Requiring lower level control access, the SDR approach involves injecting phase noise directly into the control lines. As a result, the sampling time of the noise is not necessarily restricted by the gate time $t_{G}$. However, even in the case where the sampling time is set by $t_{G}$, the injected noise and gates are inherently asynchronous; thus, phase updates may frequently occur during pulses. Because the engineered noise in the SDR-based noise injection technique is produced independently of the control pulses, this method allows a more direct comparison between the engineered and measured noise via a classical signal analyzer, a method that cannot be leveraged in the gate-based approach. In addition to these distinct features, we elaborate on other facets of the SDR approach in the discussion section.

\section{Noise injection validation}

The SchWARMA noise injection protocol is validated via QNS. We utilize a set of deterministically generated fixed total time pulse sequences (FTTPSs) designed to probe the noise spectrum across a frequency band of interest; see Appendix D for further details. Each sequence $S_{k}$ is comprised of $\pi$ pulses that produce a unique filter function $g_{k}$ that is well-concentrated in frequency space [Fig. 2(a)]. The FTTPSs probe the noise spectrum by altering the number of pulses within a fixed total sequence time. This protocol is distinct from the Carr-Purcell-Meiboom-Gill-based (CPMG-based) QNS protocol [28] that probes the noise spectrum by varying the interpulse delay (and total sequence time) using a fixed number of $\pi$ pulses and it is distinct from other fixed total time sequences found from a random search [4]. Further details on FTTPS are provided in Appendix D.

Dephasing noise [Fig. 2(b)] is injected into a single qubit system via the SDR or gate-based SchWARMA procedure, thereby simulating temporally correlated noise during the FTTPS evolution. Survival probabilities are then measured for the FTTPS [Fig. 2(c)], where the survival probability for each probe sequence exhibits a distinct (and sharp) dependence on noise of specific frequencies. The resulting survival probabilities are used to construct an estimated noise spectrum [Fig. 2(d)]. Note that we define the survival probability as the probability of the qubit being in a desired state at the end of a probe FTTPS.

Below, we demonstrate that QNS reconstructions agree with the various SchWARMA-generated and injected-noise spectra. In addition, we show that SchWARMA can be used to achieve a detailed prediction of the survival probabilities 

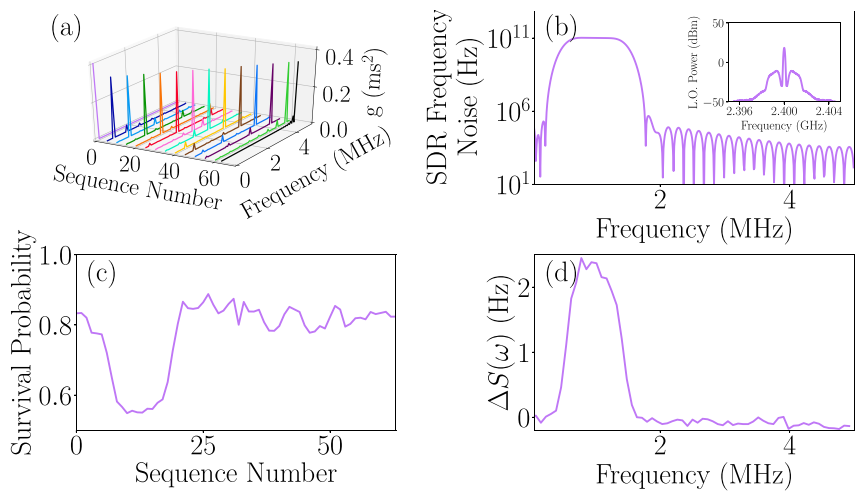

FIG. 2. SchWARMA-based noise injection and reconstruction. (a) The filter functions of the fixed total-time pulse sequences have sharp and unique dependencies on noise of different frequencies. (b) Noise of a desired spectrum, in this example bandpass, is generated with either the gate-based or SDR-based injection method. Inset: A measurement of the frequency spectrum of the $2.4 \mathrm{GHz}$ noisy carrier signal out of the SDR. This signal is mixed with an ideal control signal and upconverted to the qubit frequency. (c) The survival probabilities for each sequence can be analyzed to reconstruct the noise influencing the system (d).

resulting from the FTTPS. To separate injected noise from the native background, the native noise spectrum $S_{\text {nat }}(\omega)$ is measured using the procedure described above and subtracted from the injected noise spectrum reconstructions $S_{\text {inj }}(\omega)$ to obtain $\Delta S(\omega)=S_{\mathrm{inj}}(\omega)-S_{\text {nat }}(\omega)$.

\section{EXPERIMENTAL IMPLEMENTATION}

\section{A. Gate-based noise injection}

Gate-based noise injection provides a means for examining quantum algorithms in the presence of engineered noise when hardware access is limited to particular gate operations. Here, we demonstrate gate-based noise injection via SchWARMA. The IBMQE serves as a restricted access test bed, where we intentionally utilize the circuit-level interface to limit circuit operations to the standard IBM gate set [32], demonstrating the availability of the approach to the general user community. Phase noise is introduced through $U_{1}(\lambda)$ phase gates that are interleaved with the gates that comprise the sequences that define the probe (FTTPS). The time resolution of the noise is set by the $X$ gate timing, given by the $U(\theta, \phi, \lambda)$ gate time. The $U$ gate implements generic unitary operations using three configurable frame changes interleaved with $R_{x}(\pi / 2)$ and $R_{x}(-\pi / 2)$ rotations. The frame changes are used to set the phases $\theta, \phi, \lambda$.

Employing the five-qubit (T-shaped) Vigo processor for our experiment [33], where $t_{G} \approx 70 \mathrm{~ns}$, we prepare a single qubit in the equal superposition state, apply the FTTPS with injected phase noise, apply a gate that would return the state to $|0\rangle$ in the absence of noise, and measure. The experiment is performed over 50 independent realizations of SchWARMA trajectories for each probe sequence, where statistics for each experiment are collected over 1000 shots. The complete noise injection and noise spectrum estimation experiment is conducted over five calibration cycles and bootstrapped to mitigate spectral anomalies solely due to variations in hardware characteristics across calibration cycles, thereby improving spectral estimates. Refer to Appendix E for further information regarding optimized experimental practices and procedures.

Through the SchWARMA gate-based injected noise protocol, we find good agreement between the desired and reconstructed noise spectra. In Fig. 3, we show the results for three distinct classes of noise spectra: (a) a single bandpass spectrum of varying width, (b) a multibandpass spectrum with varying center frequencies, and (c) $1 / f^{\alpha}$ noise. We consider single bandpass bandwidths of $\Delta \omega=0.18,0.54,0.89 \mathrm{MHz}$, with a center frequency of $\omega_{0}=1.43 \mathrm{MHz}$. We explore the versatility of SchWARMA by separating the injected noise into two bandpass regions centered at $\omega_{0}=1.07 \mathrm{MHz}$ and $\omega_{1}=1.79 \mathrm{MHz}$ and subsequently, $\omega_{0}=0.64 \mathrm{MHz}$ and $\omega_{1}=$ $2.21 \mathrm{MHz}$, while keeping the bandwidth of $0.18 \mathrm{MHz}$ constant for each region. Lastly, we consider injected $1 / f^{\alpha}$-type noise with $\alpha=-2,-1,0,1,2$. Such frequency dependence is commonly found in superconducting [34-38] and semiconductor $[3,39,40]$ qubit systems.

The resulting spectral density estimates yield spectral features, such as peak height, bandwidth, and center frequency, that are found to be in good agreement with the desired noise profiles. Additional spectral features observed at high frequency have been determined to be artificial, resulting from increased gate error as higher frequency regimes are probed with sequences containing a greater number of pulses. The manifestation of gate errors in dephasing noise spectra has been previously noted and mitigated using spin-locking (SL) techniques [41]. IBM's quantum control toolbox [42] provides a means for exploring SL-based methods, as well as pulseerror compensating sequences for QNS. We focus on the latter and create a set of robust FTTPSs (RFTTPSs) composed of calibrated, true $R_{x}(\pi)$, and $R_{x}(-\pi)$ pulses. It has long been known in the NMR and dynamical decoupling communities that pulse-phase adjustments can aid in the suppression of pulse imperfection errors (e.g., over-/under-rotation errors) for pulse-based error mitigation schemes [43-46]. By constructing RFTTPSs composed of pulses of alternating phases [i.e., $R_{x}(\pi)$ and $R_{x}(-\pi)$ ], we effectively reduce the effect of pulse imperfections on the estimated spectra. RFTTPSs are employed on the APL system in the subsequent section, and an additional comparison between FTTPSs andRFTTPSs using the IBMQE is included in Appendix G. The use of RFTTPSs along with additional simulations (see Supplemental Material) support the conclusion that the observed high-frequency noise is primarily an artifact of gate error.

\section{B. Verification with SDR}

Our SDR-based approach to noise injection relies on direct hardware access. This approach is not platform agnostic and is presented as a means to validate SchWARMA gate-based noise injection by directly comparing results against a control line signal with the synthesized noise spectrum. To meet the requirements of lower-level access, we turn to the APL system to perform this comparison. 

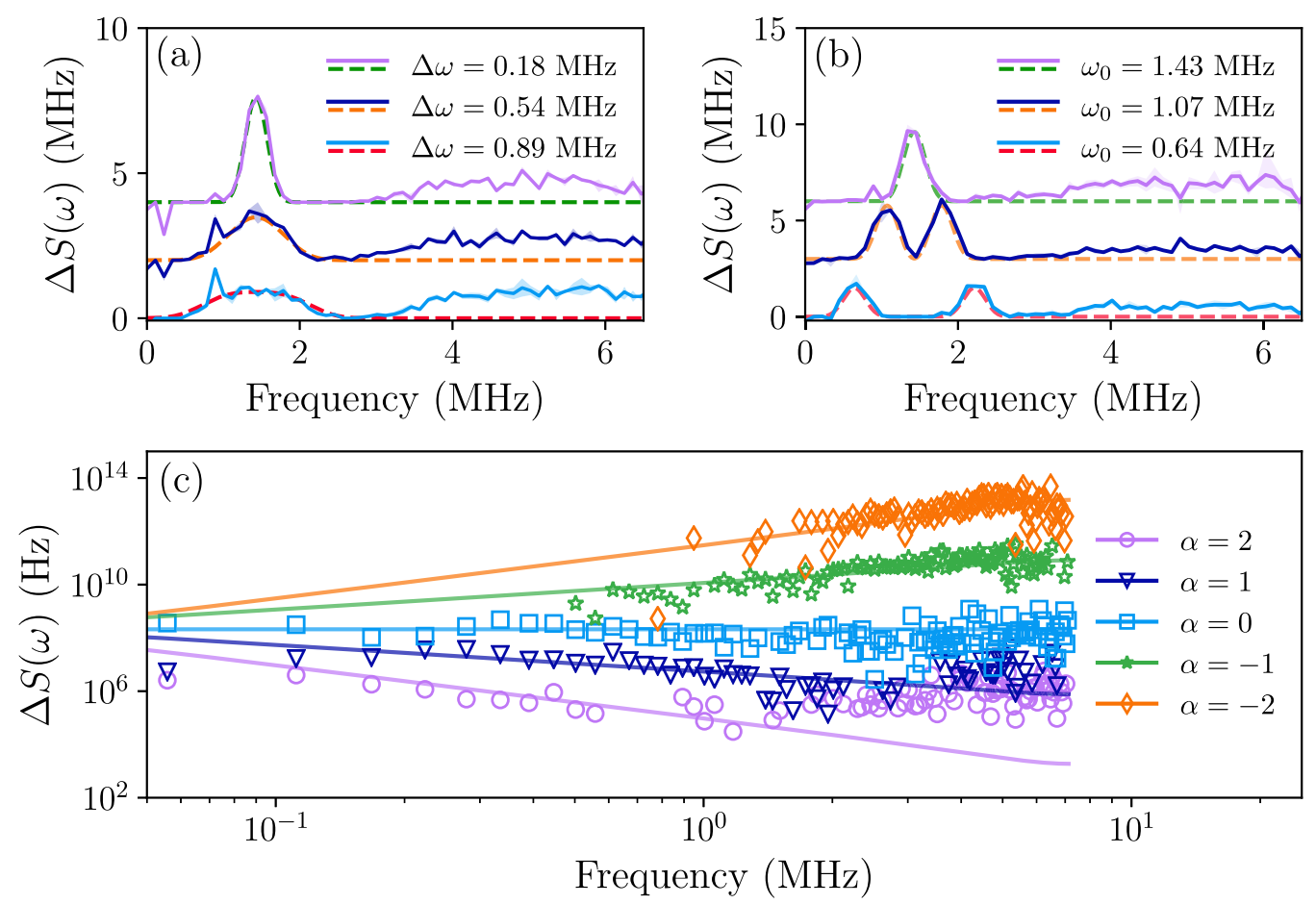

FIG. 3. SchWARMA gate-based noise injection on the IBMQE. Noise reconstructions are shown for (a) bandpass, (b) double bandpass, and (c) $1 / f^{\alpha}$ noise for different bandwidths, center frequencies, and spectral decays. Ideal spectra [dotted lines in (a) and (b) and solid lines in (c)] are found to be in good agreement with estimated spectra [solid lines in (a) and (b) and symbols in (c)]. The data shown here are compiled over five calibration cycles, with solid lines denoting bootstrapped medians and shaded regions specifying $95 \%$ confidence intervals in (a) and (b). Note that the confidence intervals are small for a majority of the estimated spectra. Data has been offset for clarity.

An SDR is configured to implement a SchWARMA model and produce $\phi_{\text {noise }}(t)$. The control signal sums the desired control phase with the phase-noise spectrum output of the SDR, acting as a faulty control signal with a drifting master clock. The SDR outputs a continuous $2.4 \mathrm{GHz}$ carrier signal with pseudorandom phase changes that are updated every 100 ns. While the phase changes may be random, one advantage of SchWARMA is that temporal correlations are permitted, meaning that the pseudorandom phase changes are biased by previously applied phase updates that mimic the dephasing error gates in the gate-based approach. In software, these fluctuations are shaped from white noise into an engineered, SchWARMA-generated noise spectrum, mimicking the gate-based approach described above. Again, the primary difference between the approaches is that the SDR-based approach interacts with the qubit asynchronously, and given that we have the same SDR-sampling rate (limited by our specific SDR-hardware and computation power) and $t_{G}$, we can use the same ARMA coefficients between them. We measure the SDR output on a signal analyzer with a $90 \mathrm{kHz}$ bandwidth as a ground-truth comparison for spectral reconstructions [Fig. 2(b) inset].

After confirmation via signal analyzer, we perform a series of RFTTPS qubit experiments on the APL system with the SDR as the master clock and the clock phase controlled by various SchWARMA models. The APL system includes a fixed-frequency transmon qubit held at $20 \mathrm{mK}$ at the mixing chamber stage of a dilution refrigerator. The qubit $(\approx 5.4 \mathrm{GHz})$ is coupled to a readout resonator $(\approx 7 \mathrm{GHz})$ and the state of the qubit is determined by dispersive measurement techniques.
See Appendices B and C for further details about SDR injection and the APL experimental setup. Experiments using the SDR approach are performed over 10,000 unique shots, and the $R_{x}( \pm \pi)$ pulses are calibrated to have $t_{G}=100 \mathrm{~ns}$ for both SDR injection and gate-based injection in the APL experimental setup. The fixed total time of noise injection experiments in the APL setup is $12.8 \mu \mathrm{s}$, well within the measured coherence times of the qubit $\left(T_{1} \approx 47 \mu\right.$ s and $T_{2} \approx$ $58 \mu \mathrm{s})$. At the start of an experiment, we apply a $R_{x}(\pi / 2)$ to initialize the qubit in $|+y\rangle$. Subsequently, an RFTTPS sequence is implemented to probe a desired frequency range. Before measurement, we apply a $R_{x}(\pi / 2)$ gate which would excite an ideally behaved qubit in the absence of noise to the $|1\rangle$ state. Using the same QNS analysis applied in the gate-based approach, we reconstruct the injected noise spectra. As is the case with the gate-based approach, the SDR injection approach can be used to produce a variety of phasenoise spectra. In Fig. 4, we show the results for (a) a single bandpass spectrum of varying width and noise power, (b) a multibandpass spectrum, and (c) $1 / f^{\alpha}$ noise. We consider single bandpass bandwidths of $\Delta \omega=0.2,1 \mathrm{MHz}$ with a center frequency $\omega_{0}=1 \mathrm{MHz}$. The double bandpass noise spectrum is engineered to have center frequencies $\omega_{0}=$ $0.5 \mathrm{MHz}$ and $\omega_{1}=1 \mathrm{MHz}$, with noise bandwidths $\Delta \omega=$ $0.5 \mathrm{MHz}$. Lastly, we consider $1 / f^{\alpha}$-type noise with $\alpha=$ $-2,-1,0,1,2$, as we did with gate-based experiments.

Reconstructed spectra are compared against the measured frequency-noise spectrum of the SDR output signal (measured on a signal analyzer) for validation. To achieve fits, the measured frequency spectra of the SDR output signal are 

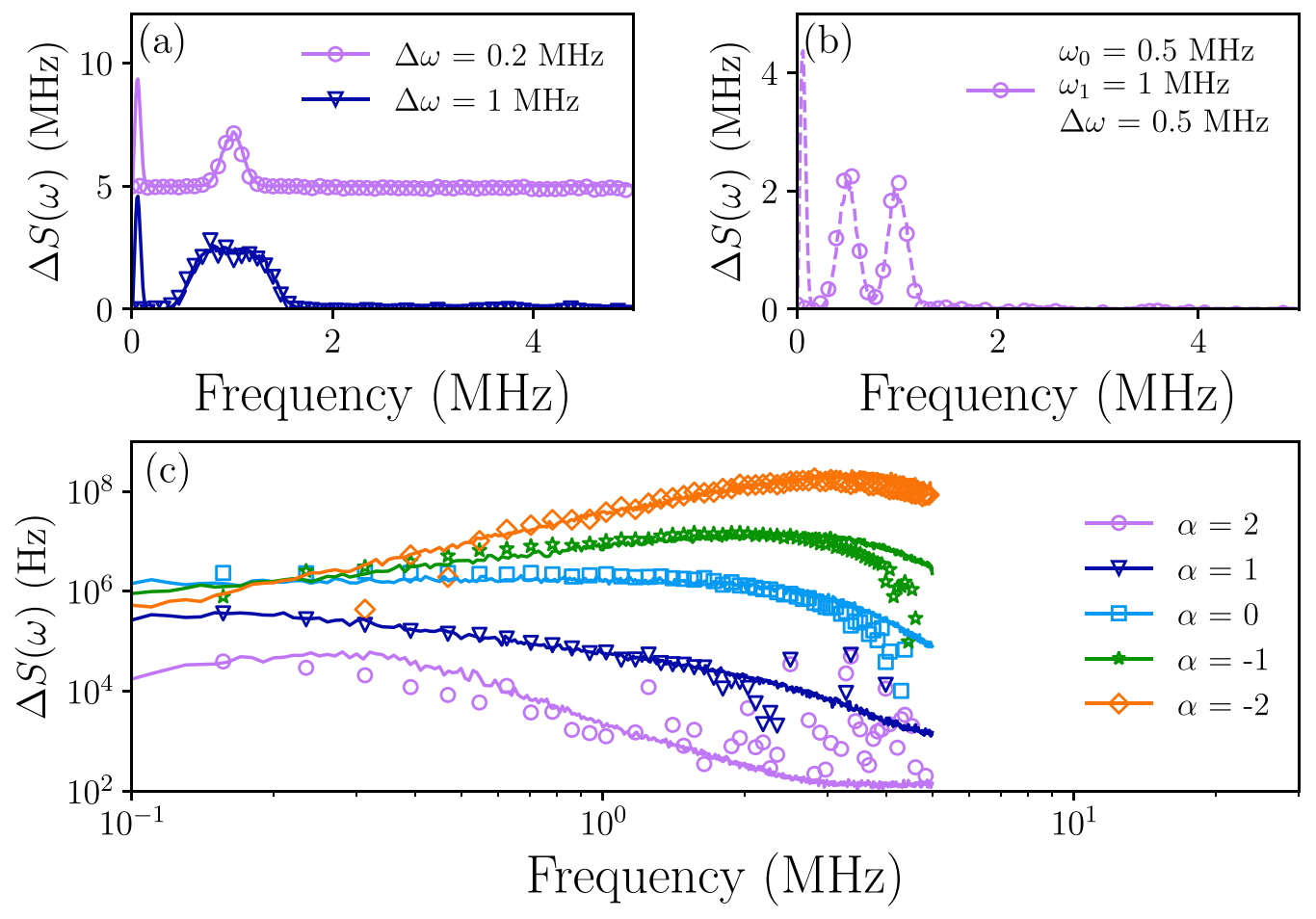

FIG. 4. Verification of SDR-based SchWARMA noise injection. Noise reconstructions are shown for (a) bandpass, (b) double bandpass, and (c) $1 / f^{\alpha}$ noise for different bandwidths, center frequencies, and spectral decays. The data shown are reconstructed from the average survival probability of 10,000 unique shots per sequence, each with a unique phase trajectory. Points represent noise reconstructions, and solid lines are measurements performed on the generated noise by a signal analyzer. In (a) and (c), plots are offset for clarity.

each multiplied by a scaling factor, constant over experimental setup, to account for attenuation of the signal between room temperature and the qubit. Overall, the reconstructed noise spectra match the injected spectra well. The measured bandwidths, peak heights, and center frequencies of the injected bandpass and double bandpass spectra are in good agreement with the injected noise spectra. The behavior of $1 / f^{\alpha}$ spectra match the expected power dependencies best within approximately $0.1 \mathrm{MHz} \leqslant \omega_{0} \leqslant 2 \mathrm{MHz}$ [Fig. 4(c)]. At frequencies above $2 \mathrm{MHz}$, the innate roll off in the SDR output distorts the injected noise, leading to inaccuracies in the reconstructed noise spectra. Despite these distortions, the overall shape of the reconstructed spectra remains in good agreement with the expected noise spectra measured at the output of the SDR (solid lines).

The combination of the signal analyzer output and the reconstructed spectra provides confidence that the SDR-based approach injects phase noise in the desired manner. We can transfer this confidence to the gate-based approach by comparing reconstructions of gate-based noise injection of identical spectra on the same device. Figure 5 shows reconstructions of gate-based SchWARMA injected noise plotted on top of nominally identical noise injected using the SDR experimental approach. Gate-based injection experiments performed on the APL experimental setup are measured over 200 unique SchWARMA trajectories, with 1000 shots per trajectory. We reiterate that both measurements in Fig. 5 are performed on the same APL experimental setup. These results show excellent agreement between the two methods, with the primary discrepancy between the approaches due to finite sampling effects on the measurements and number of SchWARMA trajectories used in the gate-based approach. Further comparisons between the gate-based and SDR approaches are included in Appendix F.

\section{SCHWARMA MODEL PREDICTION}

Much like classical ARMA, a SchWARMA model's associated power spectrum can be used to model real physical systems and predict in closed form their average response to given stimuli (here pulse sequences). This effectively closes the loop between the generation and injection processes. Here, using the known injected SchWARMA model, we fit a few ancillary parameters to experimentally generated FTTPS data. Figure 6 shows the results of this fitting process for sample experiments performed via SDR and gate-based noise injection (details of this process are found in the following paragraphs). The top panel shows SDR and gate-based data for the $1 \mathrm{MHz}$ bandpass experiments whose reconstructions are shown in Figs. 4 and 5, and the bottom panel displays gate-based data from the double bandpass experiment with $\omega_{0}=1.07 \mathrm{MHz}$ shown in Fig. 3(a). The accuracy of these fits show that SchWARMA models can be used to effectively predict the expected survival probabilities. This capability conveys that SchWARMA can be a powerful tool for parametric modeling of temporally correlated noise environments and, therefore, improved quantum system dynamics simulation informed by experimental data.

Given a FTTPS probe sequence $k$ [with $n_{k} R_{x}(\pi)$ pulses] and filter function $g_{k}$, we predict the survival probability $p_{k}$ 

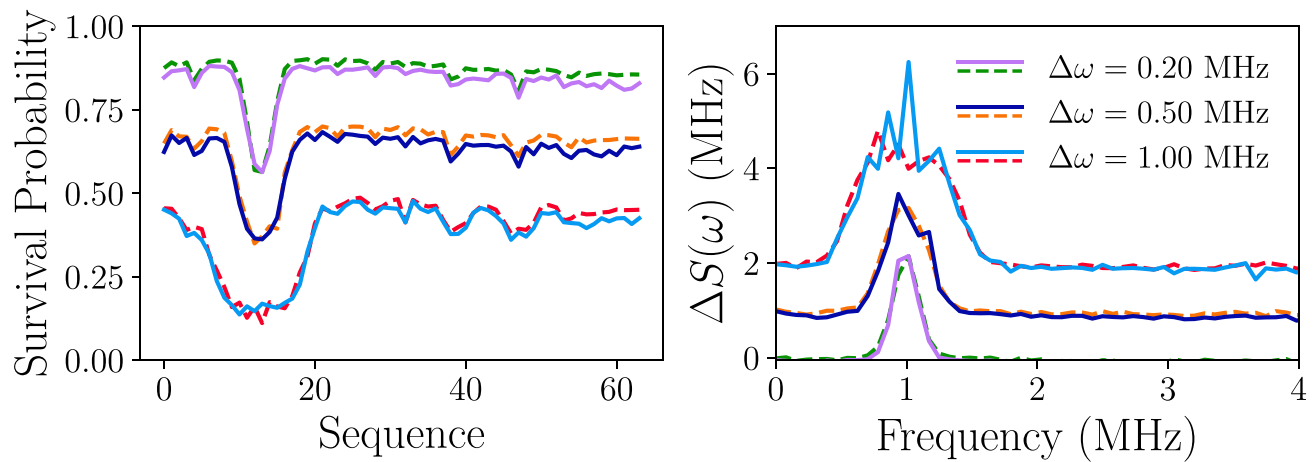

FIG. 5. Comparison between gate-based (solid lines) and SDR-based (dashed lines) SchWARMA noise injection for bandpass dephasing noise with three different bandwidths. Good agreement between the approaches is found for all spectra considered; thus, validating SchWARMA gate-based noise injection. Data for $\Delta \omega=0.5,1.0 \mathrm{MHz}$ are vertically offset by 0.2 for survival probabilities (left) and $1 \mathrm{MHz}$ for spectrum reconstructions (right).

using the model

$$
p_{k}=\frac{1}{2}+\frac{1}{2} \exp \left[-g_{k}\left(S_{\text {nat }}(\alpha)+S_{\text {inj }}\right)-c_{1} n_{k}-c_{2} n_{k}^{2}\right],
$$

where $S_{\text {nat }}(\alpha)$ is a parametric model of the native dephasing noise power spectrum (see below), $S_{\text {inj }}$ is the (known) injected spectrum and $c_{1}$ and $c_{2}$ capture the effects of stochastic (i.e., $X$ dephasing) and coherent errors on the $R_{x}(\pi)$ pulses, respectively. The pulse error terms are meant to capture the artificial high frequency features observed in the spectral estimates shown above, most notably in the case of the IBMQE results where FTTPSs without pulse-error compensation are used. We use Eq. (4) to set up a nonlinear least-squares problem to estimate the parameters $\alpha$ and $c_{i}$ using a set of FTTPSs. We further expand upon the motivation for the model and present additional results in Appendix G.

The native noise $S_{\text {nat }}$ is, in general, qubit and system dependent. For the APL system (Fig. 6 top), we found that a combination of a Lorentzian spectrum $A /\left(1+\omega^{2} / \omega_{c}^{2}\right)$, with

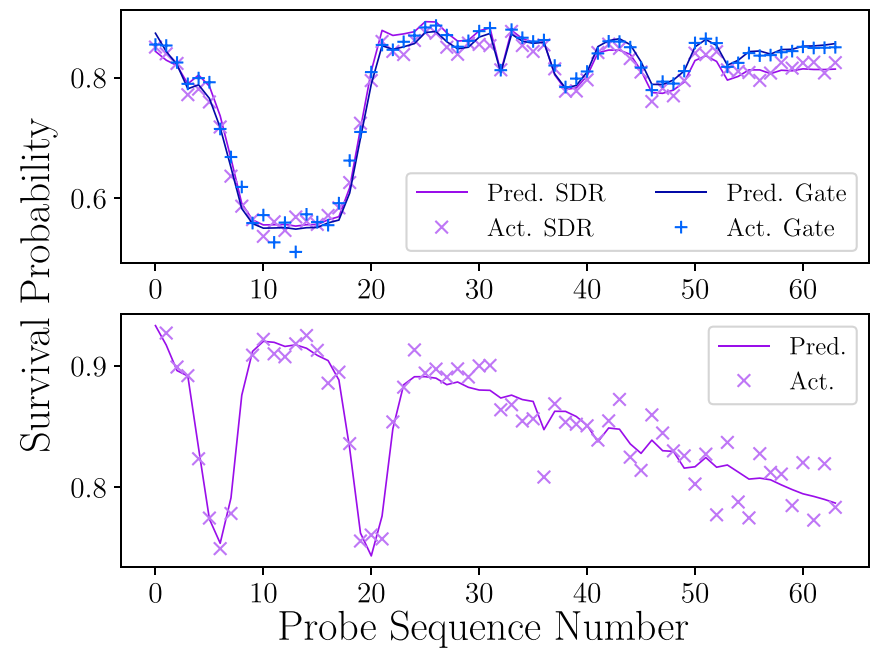

FIG. 6. Comparison of SchWARMA predicted to actual experimentally measured survival probabilities using FTTPS. Top: SDRand gate-based noise injection of a wide bandpass spectrum on the APL experimental platform. Bottom: Gate-based injection of a double bandpass spectrum on the IBMQE. unknown amplitude $A$ and cutoff frequency $\omega_{c}$, and a white noise floor with power $\sigma^{2}$ captured the observed native dephasing noise (i.e., $\alpha=\left\{A, \omega_{c}^{2}, \sigma^{2}\right\}$ ), which exhibited lowfrequency energy that was not well modeled by the white noise floor nor the control noise terms $c_{i}$. For the IBM system (Fig. 6, bottom), we found that there were isolated resonances at the zeroth and eighth sequences, and beyond that the noise was well approximated by a white noise floor. Thus, instead of introducing additional model terms in $S_{\text {nat }}$ to account for these two sequences (i.e., the native noise in those frequency bands), we ignored these sequences in the estimation (as the introduction of two more ancillary terms would perfectly match these points without changing the estimates for the unknown white noise floor power $\sigma^{2}$ and $c_{i}$ ). Again, we emphasize that Fig. 6 shows strong agreement of the least-squares fits to the measured experimental data and that we are only fitting over the ancillary background and control noise terms, not on any terms associated with the injected spectrum.

\section{DISCUSSION}

\section{A. Beyond stationary, Gaussian dephasing noise injection}

The focus of the paper presented here was on stationary, Gaussian dephasing noise with a variety of parametric spectra, but there are a number of straightforward generalizations of the gate-based approach that can be applied using the same tool set. For example, non-Gaussian noise can be introduced by applying an arbitrary nonlinearity on the output of the underlying ARMA model or by considering non-Gaussian Levy-stable distributions as inputs. Given access to arbitrary unitary gates in a system, one could look beyond $Z$-gate dephasing errors and have dephasing noise on the $X$ or $Y$ axes (or on the angle defining the axis of the control), or multiaxis dephasing errors (potentially each with its own spectrum). Nonstationary noise can be generated using heteroskedastic generalizations of ARMA (itself a potential source of $1 / f$ noise) to vary the noise power over time or by explicitly varying the terms of the SchWARMA model to produce, e.g, bandpass signals that move in frequency or drifting $\alpha$ terms in $1 / f^{\alpha}$ noise. Additionally, the gate-based approach trivially allows for the introduction of gate-dependent errors, for example, by injecting error gates only after nonidentity gates 
or mimicking multiplicative control noise by using errors that are proportional to the desired rotation angles of ideal control gates. We note that errors that move beyond the free $Z$-gate phase updates should be compiled into the ideal gates to keep the overall circuit length the same. Finally, it is clear that this approach is readily extendable to any number of qubits and, furthermore, the injected errors could be themselves spatially correlated between qubits.

\section{B. Complexity and scalability considerations for noise injection approaches}

While the versatility of the gate-based SchWARMA approach allows for noise injection on any cloud-based platform, there are some advantages to implementing the SDR approach when physical access to the hardware is available. The primary limitation of the gate-based approach is the time cost associated with uploading the circuit (for cloud systems) or arbitrary waveform generator output (for inhouse systems). Ideally, each measurement shot of the system should be generated using a different SchWARMA-generated noise trajectory, and the SDR-based approach does not require changing the underlying circuit to impart a new noise sequence. As a compromise, we use a limited number of SchWARMA noise trajectories (typically 50) and use repeated measurements of these trajectories to build up statistics. Obviously, this trade-off in time has costs in the error variance of the spectrum reconstructions which is not present in the SDR-based approach, and despite this approximation, the SDR-based experiments are still roughly $10 \times$ faster to complete than the gate-based experiments (in-house). A potential avenue to circumvent these statistical issues would be to build SchWARMA into specialized hardware in a similar fashion to randomized compilation $[47,48]$, where instead of randomly inserting Pauli frame changes for each circuit shot, SchWARMA-driven error gates are compiled into the ideal circuit in real-time for each circuit shot. Another advantage of the SDR-based approach is the potential (with more capable/additional hardware) to inject noise at frequencies much higher than the gate-based limit and explore alternative, non-gate-based spectroscopic techniques.

Beyond the requirement of physical access, there are some limitations to consider with the SDR-based approach that limits its universality. Modifications of the underlying ARMA models can be performed to produce similar extensions as in the gate-based approach, but the ability to move beyond $Z$-dephasing errors to, e.g., control amplitude errors would require additional experimental apparatuses. Furthermore, while we expect to achieve simultaneous noise injection using the SDR-based approach on a few qubits in the near future, the hardware burden limits the scalability of this approach, whereas the gate-based approach is essentially infinitely scalable to multiqubit experimentation. Again, the incorporation of SchWARMA into a randomized compilation-like approach could overcome these limitations.

\section{CONCLUSIONS}

Although quantum systems are commonly subject to temporally correlated noise, the impact of such noise processes on the performance of quantum algorithms is not well understood. Here, we present a method for engineering and injecting temporally correlated dephasing noise that can be used to elucidate key features of noise resilience and vulnerability in quantum algorithms for noise characterization, sensing, and computing. The approach enables noise injection of arbitrary noise spectra via SchWARMA, a statistical tool for modeling, estimating, and generating semiclassical noise in quantum systems. SchWARMA is used to synthesize engineered phase noise spectra that are introduced by adjusting the control master clock to emulate classical temporally correlated dephasing noise processes. We show that the experimental system dynamics generated by engineered noise can be predicted by SchWARMA-based models; therefore, closing the loop between generation and injection processes. Our results indicate that SchWARMA is a highly flexible tool for injecting engineered noise and developing experimental data-informed parametric noise models for simulating quantum system dynamics. The techniques presented here can be readily extended to multiaxis noise, non-Gaussian and nonstationary noise, and multiqubit scenarios. This points toward the generalizability and applicability of SchWARMA as a tool for understanding, evaluating, and improving quantum algorithms subjected to a wide range of temporally correlated noise processes relevant to current and future hardware platforms.

\section{ACKNOWLEDGMENTS}

The authors acknowledge JHU/APL for the infrastructure investments required to perform these experiments. In addition, the authors acknowledge Ben Palmer, Neda Foroozani, and Kevin Osborn from the Laboratory for Physical Sciences for technical support and guidance. The authors acknowledge Russell Lake, Xian Wu, and Hsiang-Sheng Ku for chip design and fabrication. G.Q. and L.T. acknowledge funding from the U.S. Department of Energy (DOE), Office of Science, Office of Advanced Scientific Computing Research (ASCR) Quantum Computing Application Teams program, under fieldwork Proposal No. ERKJ347. G.Q., K.S., and L.T. acknowledge support from ARO MURI Grant No. W911NF-18-1-0218. B.D.C. acknowledges support from DOE Office of Science ARQC Grant No. DE-SC0020316. J.L and D.P acknowledge support from the National Science Foundation (NSF) (Award No. 1839136) and the DOE through FNAL. A.M., J.E., G.Q., K.S., K.M., C.T., B.T.S., J.A.H., B.D.C., and T. S. acknowledge support from the United States Department of Defense. The views and conclusions contained in this document are those of the authors and should not be interpreted as representing the official policies, either expressly or implied, of the United States Department of Defense or the U.S. Government.

\section{APPENDIX A: CHOICE OF SPECTRA}

To emulate dephasing with a particular noise spectrum, we use a SchWARMA model to generate $Z$ gate errors that are interleaved into a quantum circuit. These errors map to noise spectra that are observable with QNS techniques. A survey of QNS experiments shows numerous noise characteristics that often follow a $1 / f^{\alpha}$ curve. However, $\alpha$ has been observed to 
TABLE I. Summary of experimentally observed native noise spectra.

\begin{tabular}{lll}
\hline \hline Ref. & \multicolumn{1}{c}{ Qubit type } & \multicolumn{1}{c}{ Noise $\alpha$} \\
\hline$[37,38]$ & superconducting flux qubit & 0.9 \\
{$[34]$} & superconducting resonators & 1 \\
{$[35,36]$} & superconducting transmon & $1,0.45,0.58,0.79$ \\
{$[39]$} & double quantum dot & $\geqslant 1$ \\
{$[3]$} & MOS quantum dot & $2.5,0.8$ \\
{$[40]$} & spin qubit & 1,2 \\
\hline \hline
\end{tabular}

vary from $\approx 0.5-2.5$ (see Table I). Furthermore, tones in the noise spectra have been observed in varied qubit platforms attributed to charge noise or two-level systems. To emphasize the power of SchWARMA, we chose to emulate various noise profiles including noise with $1 / f^{\alpha}$ for various values of $\alpha$, and single (and multi) bandpass noise. Specifics of how these spectra are generated in the SchWARMA framework and additional examples can be found in Ref. [26]. We point out that while the long-time correlations induced by $1 / f^{\alpha}$-type noise are believed to be more detrimental to circuit performance, bandpass noise may be of interest in evaluating quantum sensing protocols (e.g., Ref. [49]) and certainly for precisely evaluating QNS protocols [50].

\section{APPENDIX B: EXPERIMENTAL SETUP}

A diagram of the APL experimental setup is shown in Fig. 7. The control signal is produced by an arbitrary waveform generator and mixed with a $2.4 \mathrm{GHz}$ signal out of the SDR up to the qubit frequency. The measured device is a fixed frequency transmon qubit with a qubit frequency of $\approx 5.4 \mathrm{GHz}$ and coherence times $T_{1} \approx 47 \mu \mathrm{s}$ and $T_{2} \approx 58 \mu \mathrm{s}$. The qubit is housed in a $\mu$-metal shield under the mixing chamber stage of a dilution refrigerator, held at $20 \mathrm{mK}$. A readout resonator at $\approx 7 \mathrm{GHz}$ is coupled to the qubit, and the state of the qubit is determined by standard cQED techniques. Gate-based noise injection and SDR-based noise injection are both performed using the same experimental setup. For gate-based noise injection, the phase of the signal is controlled by the arbitrary waveform generator and the SDR outputs a pure tone. For SDR-based noise injection, phase noise is generated by the SDR. This method is discussed in further detail below.

\section{APPENDIX C: NOISE INJECTION: SOFTWARE DEFINED RADIO}

A Python interface to (GNU) radio is used to produce the phase errors that convert independent pseudorandom Gaussian variables into time-correlated Gaussian variates with the desired spectrum through a series of filters. The output of the filter is used to phase modulate the carrier wave. A graphical depiction of this process is shown in Fig. 8. An Ettus Research USRP N200, set to a $10 \mathrm{MHz}$ sampling frequency and a $2.4 \mathrm{GHz} \mathrm{LO}$, outputs a $2.4 \mathrm{GHz}$ signal with phase varying every $100 \mathrm{~ns}$. This phase-varying signal is used as a LO, mixing the control pulses, applied at an intermediate frequency, up to the qubit frequency (Fig. 7). Since the phase

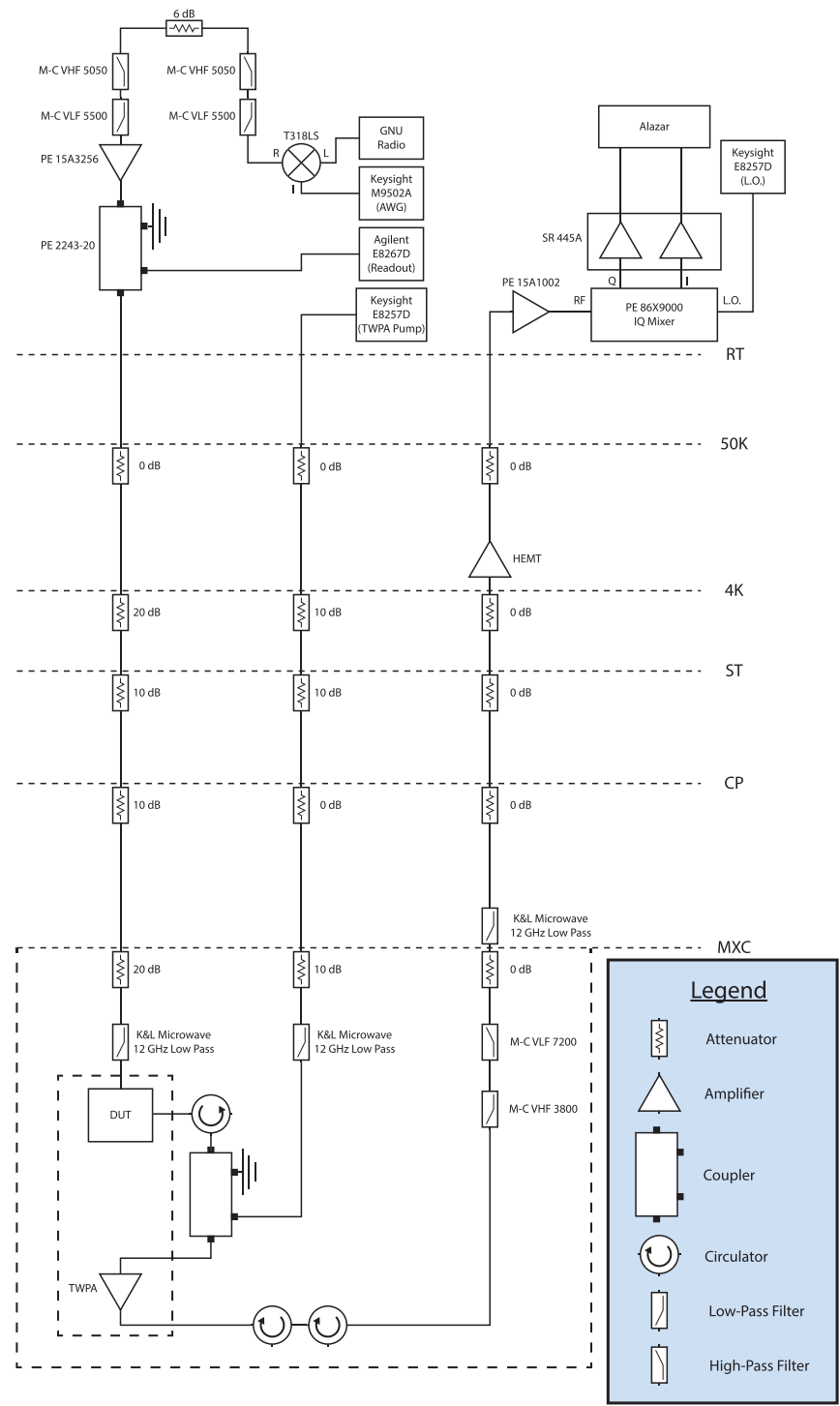

FIG. 7. Diagram of the experimental setup for noise injection experiments with the SDR on the APL experimental system.

modulation is a randomized ARMA-generated sequence, the control signals at the qubit frequency also carry the generated phase modulation in a manner identical to the underlying ARMA model in a gate-based SchWARMA approach, although we note that the phase updates in the SDR-based approach are asynchronous with the control pulses. Ultimately, this produces a control signal with a phase noise power spectrum identical to that of gate injection.

\section{APPENDIX D: FIXED-TOTAL-TIME PULSE SEQUENCES AND NOISE RECONSTRUCTION}

We reconstruct the noise spectra from survival probabilities of a set of FTTPS. A $\pi / 2$ pulse is applied to the qubit before and after each FTTPS so the qubit state sits on the equator of the Bloch sphere and is susceptible to dephasing noise throughout the duration of the pulse sequence. Within these two bookend $\pi / 2$ pulses, FTTPS experiments consist of $2^{m}$ different gate sequences, each with $2^{m+1}$ gates. The length 

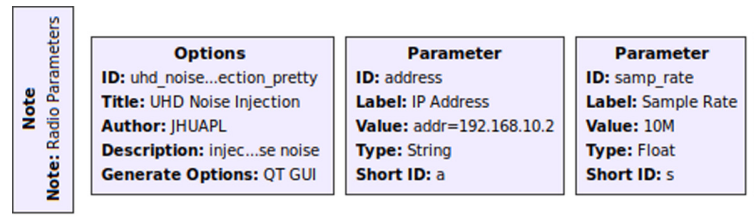

\begin{tabular}{|l|}
\multicolumn{1}{|c|}{ Parameter } \\
ID: freq \\
Label: Default Frequency \\
Value: $2.4 \mathrm{G}$ \\
Type: Float \\
Short ID: $f$ \\
\hline
\end{tabular}

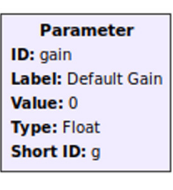

\begin{tabular}{|l|}
\multicolumn{1}{|c|}{ QT GUI Range } \\
ID: tun_freq \\
Label: UHD Freq (Hz) \\
Default Value: $2.4 \mathrm{G}$ \\
Start: $2.2 \mathrm{G}$ \\
Stop: $2.6 \mathrm{G}$ \\
Step: 1
\end{tabular}

\begin{tabular}{l}
\multicolumn{1}{c|}{ QT GUI Range } \\
ID: ampl \\
Label: Envelope Amplitude \\
Default Value: $90 \mathrm{~m}$ \\
Start: 0 \\
Stop: 1 \\
Step: $100 \mathrm{u}$ \\
\hline
\end{tabular}
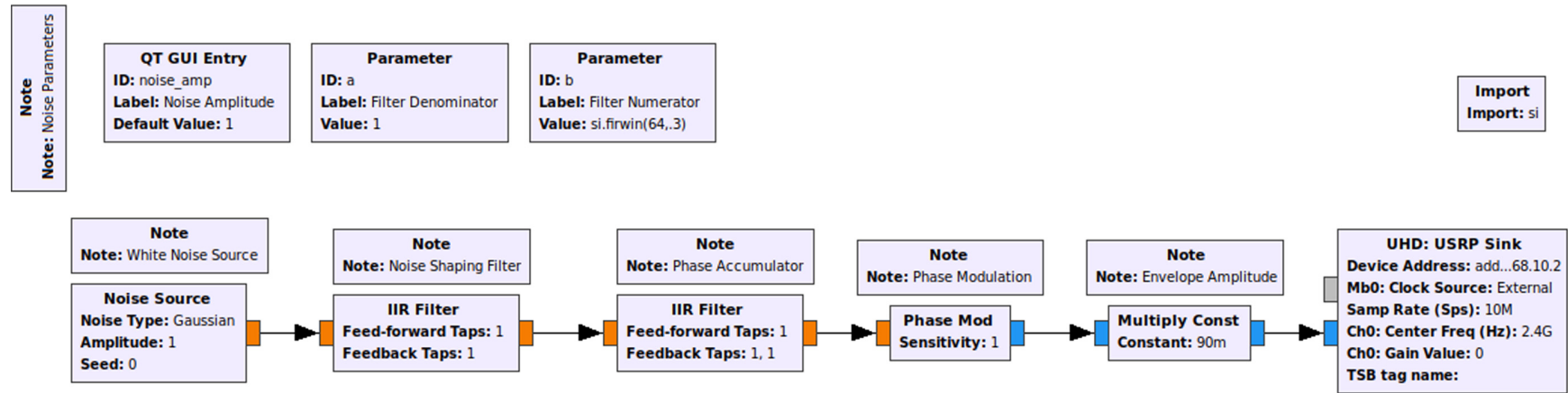

FIG. 8. Example GNU Radio flow graph for bandpass noise. The parameters of the noise shaping filter block determine the power spectrum of the injected noise.

of each gate, $t_{\text {gate }}$, is held constant. This gives a fixed total time of $\left(t_{\text {gate }} 2^{m+1}\right)$ for each sequence. For experiments on the APL system, we typically used $t_{\text {gate }}=100 \mathrm{~ns}$ and $m=6$ for a total control duration of $12.8 \mu \mathrm{s}$. Each gate in these sequences is either an identity gate or a $\pm \pi$ pulse. The sign changes of the function $\operatorname{sgn}\left\{\cos \left(\pi n k / 2^{m}\right)\right\}$ where sgn is the signum function and $k$ is the sequence number (ranging here from 0-63) determine which pulses $n$ (here ranging from $0-127)$ are $\pi$ pulses. The remaining pulses are identity gates. In Fig. 9, we illustrate the gate sequences applied for $n=$ $0,3,30$, and 60 . The motivation behind these sequences is that single-axis $\pi$ pulses should create a so-called modulation function whose values are \pm 1 , and this particular set of sequences should have good frequency concentration and little overlap.

Noise reconstruction is performed by implementing nonnegative least-squares regression to solve for the noise $S$ which gives a minimum to $\|\Phi S-\chi\|_{2}$, where $\Phi$ is a matrix whose rows sample the controls sequences' filter functions at discrete points. For the FTTPS used here, we have an excellent condition number for $\Phi$ of $\approx 1.63$, indicating that this regression problem is very well-posed. The filter function is related to the squared Fourier transform of the pulse sequence (assuming instantaneous pulses), and $\chi$ is related to survival probability $p$ by $p=1 / 2+1 / 2 e^{-\chi}$.

Fixed total time gate sequences have a constant $T_{1}$ dependence, avoid long-time steady state values, and produce better-conditioned matrices for inversion than traditional CMPG sequences [28]. The primary disadvantage of this method is that the highest frequency for reconstruction analysis is limited to $2 / t_{\text {gate }}$, which for the IBM system used is $7.14 \mathrm{MHz}$ and for the in-house system is $5 \mathrm{MHz}$. However, higher frequencies can be addressed with other techniques such as SL measurements [41].

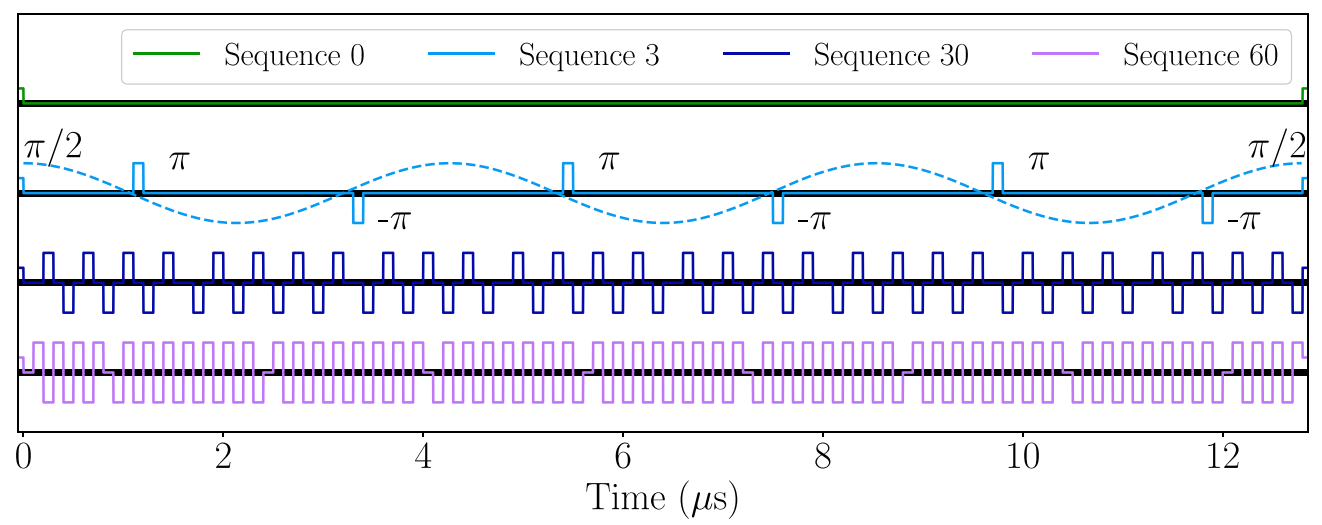

FIG. 9. Robust fixed total time pulse sequences $0,3,30$, and 60 , with $m=6$. Each sequence consists of $2^{m+1}=128$ pulses, bookended by $\pi / 2$ pulses. The sequences are composed of equally timed (100 ns for in-house experiments) identity, or $\pm \pi$ gates. For the zeroth sequence, all pulses between the two bookend $\pi / 2$ pulses are identity gates. Subsequent sequences have increasing number of $\pm \pi$ pulses. The $+\pi$ gates are represented by positive square pulses, $-\pi$ gates are represented by negative square pulses. Zeros represent identity gates. Note that zero for each sequence is offset for clarity. Sequence 3 is annotated for clarity. The function $\cos (3 \pi n / 64)$ determines the placement of $\pm \pi$ gates in the sequence. Each gate following a zero of this function is a $\pi$ gate. 


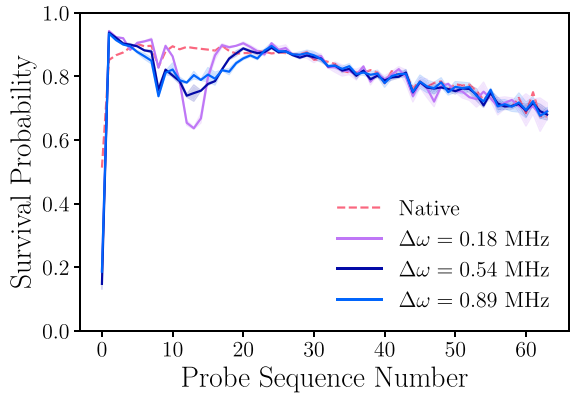

(a)

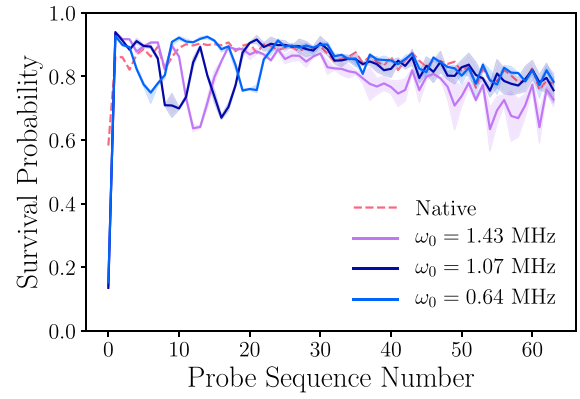

(b)

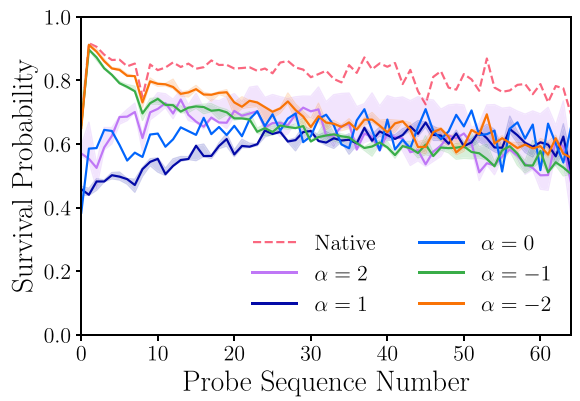

(c)

FIG. 10. Survival probabilities for injected noise experiments on the IBM quantum experience. (a) Bandpass noise injection survival probabilities shown for each bandwidth considered. (b) Double bandpass injection survival probabilities for different center frequencies $\omega_{0}$ and $\omega_{1}$, where $\omega_{0}$ denotes the peak closest to probe sequence zero. (c) $1 / f^{\alpha}$ noise injection survival probabilities for different $\alpha$ values. Note that in all subfigures, the native noise survival probability is denoted by the dashed line.

\section{APPENDIX E: EXPERIMENTAL PRACTICES FOR IBM EXPERIMENTS}

There are a number of experimental practices that we enforce to establish accurate comparisons and evaluations of native and injected noise spectra. Perhaps the most notable feature of the IBM quantum experience is the daily calibration, where qubit transition frequencies are measured and single and multiqubit pulses are calibrated. We observed that qubit performance can vary significantly between, and even within, calibration cycles. Calibration errors can appear as large shifts in qubit survival probability when a suite of experiments are performed across a calibration. Furthermore, subtle changes in survival probabilities are observed, depending upon when one performs the experiments, e.g., experiments performed soon after a calibration can be quite distinct from those performed close to the next calibration due; this is likely due to unwanted drifts in qubit frequency and the control master clock.

To mitigate the effects of calibration for the large suite of experiments required to obtain the data shown in the main text, we enforce the following set of experimental practices. Let $C_{j}$ be a particular comparison which contains experiments for a set of FTTPSs used to probe the native and injected noise spectra; let $S_{j}$ denote the individual probe sequence experiment. We require each $C_{j}$ to be performed within a calibration cycle and then collect each $C_{j}$ for five distinct calibration cycles to capture typical hardware behavior. For a comparison $C_{j}$, the ordering of $S_{j}$ is randomized to average out shifts in survival probability due to qubit drift within a calibration cycle. We further attempt to mitigate the effects of qubit drift by performing $S_{j}$ for both the native and injected noise experiments back-to-back. This last procedure allows for a direct comparison between native and injected noise spectra each FTTPS and, thus, each frequency bin. Together, these practices provide confidence in the capability of SchWARMA noise injection as a universal noise injection technique.

\section{APPENDIX F: NOISE INJECTION SURVIVAL PROBABILITIES}

Noise spectrum reconstructions shown in the main text are calculated based on survival probabilities obtained from
FTTPS experiments performed with and without injected noise. In Fig. 10, the survival probabilities for each of the gate-based noise injection experiments performed on the IBM quantum experience are shown as a function of probe sequence number. Note that this particular point of view offers a similar, yet inverted, representation of the noise spectral density due to the relationship between probe sequence number and probing frequency. More concretely, the increasing probe sequence index directly corresponds to an increasing number of $\pi$ pulses, which in turn corresponds to shifting the location of the fundamental harmonic of the probing sequence filter function to higher frequency. Thus, one can observe native or injected noise spectral features from the survival probabilities without performing an inversion or least-squares optimization. Furthermore, an attractive feature of examining the survival probability versus the spectral estimate is that the former does not amplify the effect of spurious spectral features. In many scenarios, the injected noise spectra are found to be in closer agreement with the desired spectra from the perspective of the survival probability.

In Fig. 11, we present the survival probabilities associated with noise reconstructions measured on the in-house experimental setup that are presented in the main text. The native noise background (red dashed lines) was measured once, while measurements of injected spectra were taken over many days. We do not account for changes over time in the native noise background apparent in the high probe sequence numbers of Fig. 11(d). Figures 11(a)-11(c) correspond to survival probabilities using the SDR injection method, while Fig. 11(d) corresponds to gate-based injection experiments.

\section{APPENDIX G: EFFECT OF GATE ERROR ON NOISE SPECTRUM ESTIMATION}

Pulse-error compensating probe sequences dramatically reduce artificial spectral features at high frequency. Here, we provide support for this statement by examining the effect of pulse error on FTTPS noise reconstruction from both a theoretical and experimental perspective. The theoretical analysis relies on previous results from Ref. [51] to examine the effect of pulse error on survival probability. Subsequent experiments focus on the comparison between FTTPS and its robust counterpart on the IBMQE. 


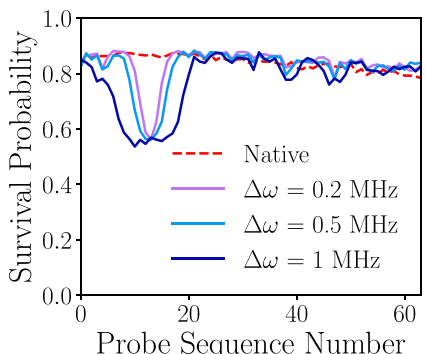

(a)

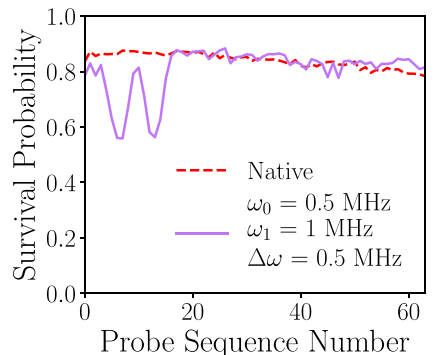

(b)

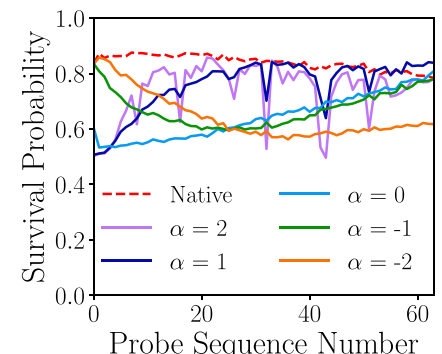

(c)

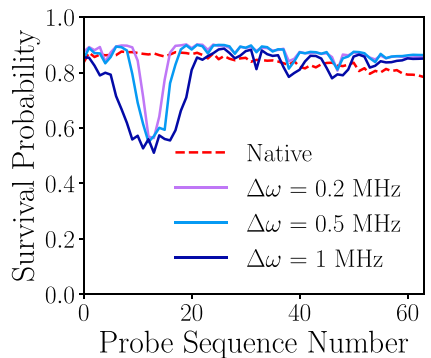

(d)

FIG. 11. Survival probabilities for injected noise experiments on the in-house setup. (a) Bandpass noise injection survival probabilities using the SDR injection method. The two curves with $\Delta \omega=0.2 \mathrm{MHz}$ correspond to differing noise powers, as shown in the main text. (b) Double bandpass injection survival probabilities using the SDR injection method. (c) $1 / f^{\alpha}$ noise injection survival probabilities for different $\alpha$ values using the SDR injection method. (d) Bandpass noise injection survival probabilities using the gate-based injection method.

\section{Theoretical analysis}

We model the system as a single qubit subject to semiclassical dephasing noise and multiplicative control noise. Assuming only amplitude control, the control Hamiltonian is given by

$$
H_{C}(t)=\frac{\Omega(t)}{2} \sigma^{X},
$$

while the error Hamiltonian

$$
H_{E}(t)=\frac{\Omega(t)}{2} \epsilon(t) \sigma^{X}+\frac{\beta(t)}{2} \sigma^{Z}
$$

explicitly defines the noise contributions. In $H_{E}(t)$, the control amplitude $\Omega(t)$ is augmented by an error function

\section{Coherent Errors}
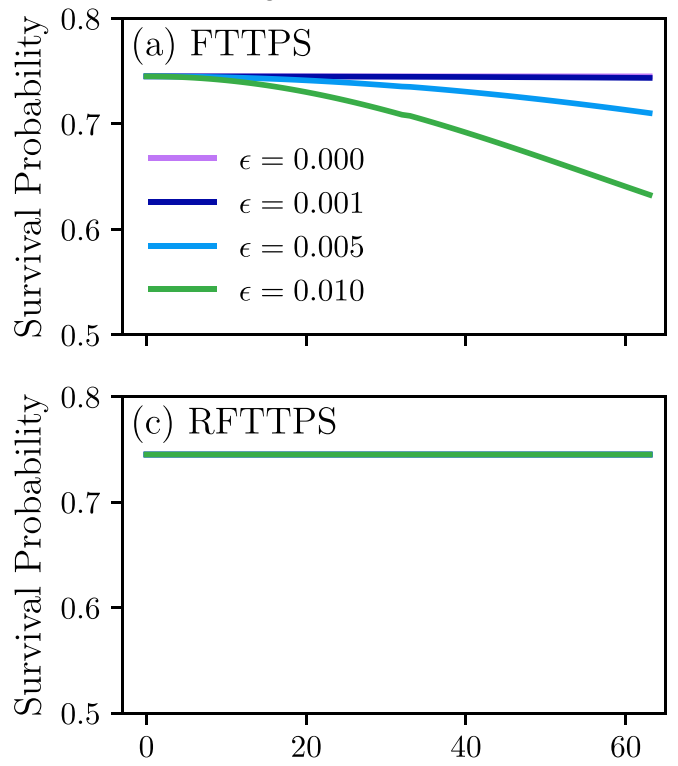

Probe Sequence Number $\epsilon(t)$ that dictates the characteristics of the control noise. Both $\epsilon(t)$ and $\beta(t)$ are assumed to be wide-sense stationary, Gaussian random variables, with means $\overline{\epsilon(t)}$ and $\overline{\beta(t)}$ and two-point correlation functions $C_{\epsilon}(\tau)=\overline{\epsilon(\tau) \epsilon(0)}$ and $C_{\beta}(\tau)=\overline{\beta(\tau) \beta(0)}$, respectively; $\cdots$ denotes classical ensemble averaging.

We consider the weak noise limit and examine the dynamics of $H(t)=H_{C}(t)+H_{E}(t)$ via time-dependent perturbation theory. This is accomplished by moving into the rotating frame with respect to the control Hamiltonian such that

$$
\begin{aligned}
\tilde{H}_{E}(t) & =U_{C}^{\dagger}(t) H_{E}(t) U_{C}(t) \\
& =\frac{\Omega(t)}{2} \epsilon(t) \sigma^{X}+\frac{\beta(t)}{2} f(t) \sigma^{Z} .
\end{aligned}
$$
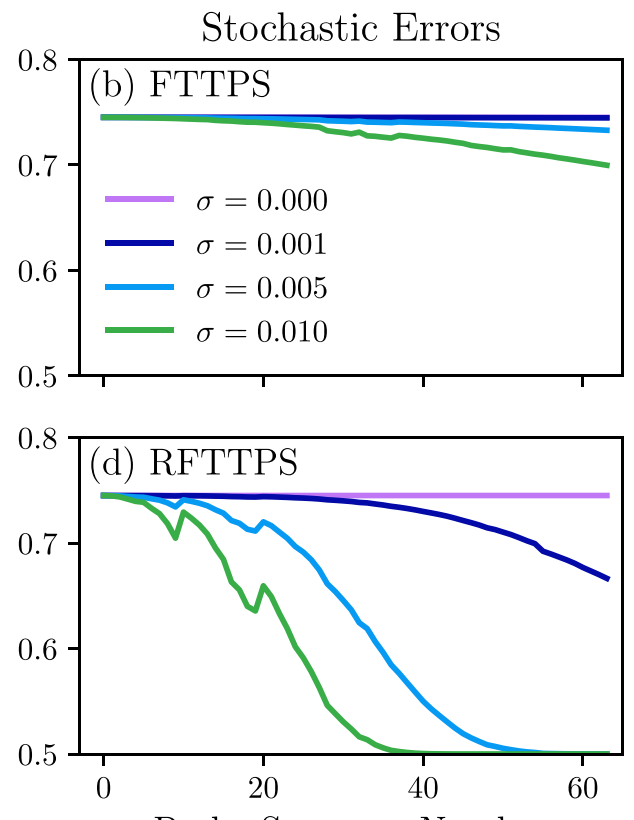

Probe Sequence Number

FIG. 12. Approximate analytical survival probabilities derived via the filter function formalism assuming a single qubit system with multiplicative control noise and dephasing. Results for coherent control errors are shown in the first column for (a) FTTPS and (c) RFTTPS. The second column displays results for uncorrelated stochastic control noise for FTTPS and RFTTPS in (b) and (d), respectively. While coherent errors appear to induce larger artificial spectral features at higher frequency than stochastic errors, they are easily corrected by the alternating phases used in RFTTPS. In contrast, RFTTPS is not effective against stochastic errors, appearing to enhanced the contribution of the control noise as opposed to reducing it. 
The control noise term commutes with the control while the dephasing term does not. As a result, the dephasing term undergoes a rotation that is dictated by the specifications of $\Omega(t)$. We consider the case where $\Omega(t)$ defines a sequence of instantaneous pulses and, thus, the dephasing term in the rotating frame reduces to its original form with an additional multiplicative function $f(t)$. Commonly referred to as the switching function, $f(t)= \pm 1$ under the conditions we have defined. Note that the FTTPSs are composed of either identity or $R_{x}(\pi)$ gates, hence, our focus on pulse sequences. The additional assumption of instantaneous pulses focuses the analysis on the effect of imperfections in the control amplitude rather than the effects of finite pulse width. While both contribute to the dynamics of the system in practice, we argue that the amplitude error is the dominant source of pulse error that contributes to the spectral features observed at high frequency in the FTTPS reconstructions.

Spectrum estimates are calculated via survival probabilities generated by initializing the qubit in $\left|+_{y}\right\rangle$, implementing a sequence of pulses to probe the environment spectrum, and measuring $\left|+_{y}\right\rangle$. More concretely, our experiments measured

$$
P(+y, t)=\overline{\left|\left\langle+_{y}|U(t)|+{ }_{y}\right\rangle\right|^{2}},
$$

where $U(t)=\mathcal{T}_{+} \exp \left[-i \int_{0}^{t} H(s) d s\right]$ is the time propagator for the system, with $\mathcal{T}_{+}$designating the time-ordering operator. In the rotating frame, $U(t)=U_{C}(t) \tilde{U}_{E}(t)$ is factorized into a propagator corresponding to the ideal control Hamiltonian, $U_{C}(t)=\mathcal{T}_{+} \exp \left[-i \int_{0}^{t} H_{C}(s) d s\right]$, and an error propagator, $\tilde{U}_{E}(t)=\mathcal{T}_{+} \exp \left[-i \int_{0}^{t} \tilde{H}_{E}(s) d s\right]$. Following Ref. [51], we parametrize the error dynamics in terms of a real, time-dependent error vector $\vec{a}(t) \equiv\left(a_{X}(t), a_{Y}(t), a_{Z}(t)\right)$, such that

$$
\tilde{U}_{E}(t)=e^{-i \vec{a}(t) \cdot \vec{\sigma}},
$$

with $\vec{\sigma}=\left(\sigma^{\mathrm{X}}, \sigma^{\mathrm{Y}}, \sigma^{\mathrm{Z}}\right)$. Typically, the error vector is expressed in terms of a perturbative magnus expansion, where $\vec{a}(t)=\sum_{k=0}^{\infty} \vec{a}^{(k)}(t)$. For sufficiently weak noise and short timescales, the expansion can be truncated to first order such that $\vec{a}(t) \approx \vec{a}^{(1)}(t)$, where

$$
a_{\mu}^{(1)}(t)=\frac{1}{2} \operatorname{Tr}\left[\sigma^{\mu} \int_{0}^{t} \tilde{H}_{E}(s) d s\right] .
$$

The approximate dynamics of $\tilde{U}_{E}(t)$ combined with $U_{C}(t)$ lead to two distinct survival probabilites for the FTTPS sequences. FTTPSs of total time $T$ generate $U_{C}(T)=I$ for the first $N / 2$ sequences or $U_{C}(T)=-i \sigma^{\mathrm{X}}$ for the second half. Combining the first-order approximation of the error dynamics with the two possible ideal control evolutions, we obtain two approximate expressions for the survival probabilities of the FTTPS sequences:

$$
P(+y \mid T) \approx 1-\overline{\left[a_{X}^{(1)}(t)\right]^{2}}-\overline{\left[a_{Z}^{(1)}(t)\right]^{2}}
$$

and $P(-y \mid T)=1-P(+y \mid T)$. These expressions exhibit dependence only on the $x$ and $z$ components of the error vector. Note that in our model, the $x$ component corresponds to the control noise, while the $z$ component captures the dephasing contribution.
Moving to the frequency domain, we obtain a relationship between filter functions and the noise spectral densities. In particular, it can be shown that

$$
\begin{aligned}
& \overline{\left[a_{X}^{(1)}(T)\right]^{2}}=\frac{1}{2 \pi} \int_{-\infty}^{\infty} F_{X}(\omega, T) S_{\epsilon}(\omega) d \omega, \\
& \overline{\left[a_{Z}^{(1)}(T)\right]^{2}}=\frac{1}{2 \pi} \int_{-\infty}^{\infty} F_{Z}(\omega, T) S_{\beta}(\omega) d \omega,
\end{aligned}
$$

where the filter functions are

$$
\begin{aligned}
& F_{X}(\omega, T)=\frac{1}{4}\left|\int_{0}^{T} \Omega(t) e^{i \omega t}\right|^{2}, \\
& F_{Z}(\omega, T)=\frac{1}{4}\left|\int_{0}^{T} f(t) e^{i \omega t}\right|^{2},
\end{aligned}
$$

and the $S_{\mu}(\omega)=\int_{-\infty}^{\infty} C_{\mu}(\tau) e^{-i \omega \tau} d \tau, \mu=\epsilon, \beta$ denotes the power spectral density for the control and dephasing noise, respectively.

Here, we consider two forms of control noise: coherent errors, where $\overline{\epsilon(t)}=\epsilon$ and $S_{\epsilon}(\tau)=\epsilon^{2} \delta(\omega)$, and zero-mean stochastic errors characterized by white noise with cutoff frequency $\omega_{c}$, i.e., $\overline{\epsilon(t)}=0$ and $S_{\epsilon}(\tau)=\sigma^{2}\left[\Theta\left(\omega+\omega_{c}\right)-\right.$ $\left.\Theta\left(\omega-\omega_{c}\right)\right]$. In the case of instantaneous pulses, we find

$$
\overline{\left[a_{X}^{(1)}(t)\right]^{2}}= \begin{cases}\frac{\epsilon^{2} \pi}{8} M^{2}, & \text { coherent } \\ \frac{\sigma^{2} \omega_{c} \pi}{4} \sum_{i, j} \operatorname{sinc}\left[\omega_{c}\left(s_{i}-s_{j}\right)\right], & \text { stochastic, }\end{cases}
$$

where $M$ corresponds to the total number of $R_{x}(\pi)$ pulses and $s_{j}$ is the location of the $j$ th $R_{x}(\pi)$ pulse in the sequence. The above expressions can be augmented to account for phase flips in the pulses, e.g., in such cases as RFTTPSs, by considering $\Omega(t) \mapsto \Omega(t) \alpha(t)$. The phase flipping function $\alpha(t)= \pm 1$, where the function's value is determined by whether the pulse sequence implements an $R_{x}(\pi)$ or $R_{x}(-\pi)$, respectively, at time $t$. When phase flips are considered, the resulting error vector component for coherent errors is

$$
\overline{\left[a_{X}^{(1)}(t)\right]^{2}}=\frac{\epsilon^{2} \pi}{8}\left(\sum_{j} \alpha\left(s_{j}\right)\right)^{2}
$$

and

$$
\overline{\left[a_{X}^{(1)}(t)\right]^{2}}=\frac{\sigma^{2} \omega_{c} \pi}{4} \sum_{i, j} \alpha\left(s_{i}\right) \alpha\left(s_{j}\right) \operatorname{sinc}\left[\omega_{c}\left(s_{i}-s_{j}\right)\right]
$$

for stochastic errors. As expected, in the coherent error case, phase-flip modulation results in a pulse error that is determined in part by the accumulated sum of the phases. While a similar quadratic dependence on the accumulated phase is observed in the stochastic error case, the expressions are more complex and reliant on interactions between phases at distinct points in time.

In Fig. 12, we show comparisons of survival probabilities for FTTPSs and RFTTPSs based on the analytical expressions derived above. We consider zero-mean, uncorrelated dephasing noise such that $\overline{\beta(t)}=0$ and $C_{\beta}(\tau)=\Gamma^{2} \delta(\tau)$. In the first column, results are shown for coherent errors for different magnitudes of the error $\epsilon$. In the second column, we show results for uncorrelated, white cutoff control noise with 


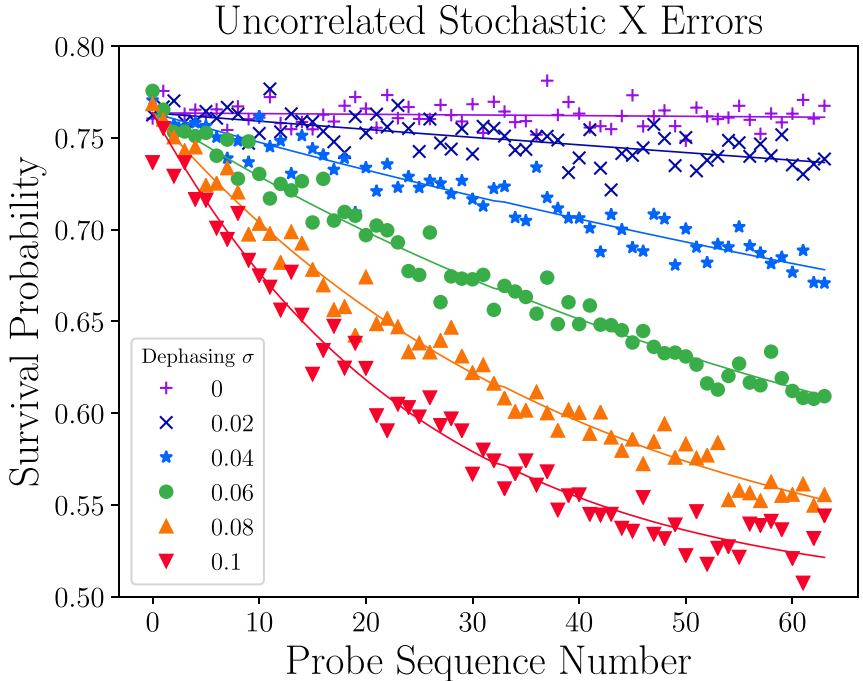

(a)

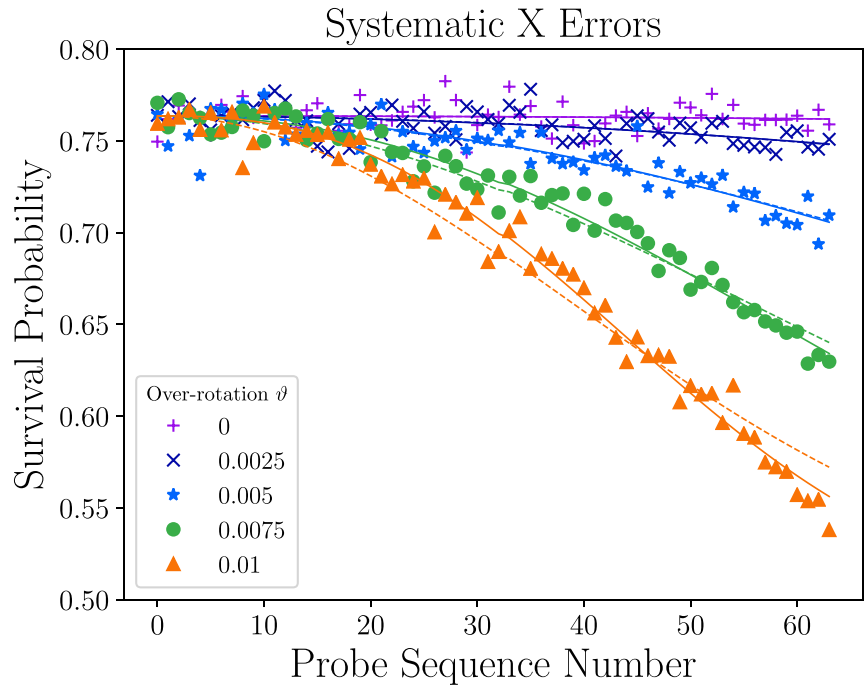

(b)

FIG. 13. Effects of errors on $R_{x}(\theta)$ gates in simulated FTTPSs in the presence of a white-noise $Z$-dephasing error. Markers indicate averages of 1000 Monte Carlo runs of SchWARMA-driven noisy circuits, with lines showing regression fits using the gate-error penalty terms. (a) Ideal $R_{x}(\theta)$ gates are followed by uncorrelated $R_{x}(\vartheta)$ where $\vartheta$ are zero mean normally distributed with standard deviation $\sigma$, resulting in $X$-dephasing errors on the $R_{x}$ gates, when averaged. The solid lines show least-squares fits of the survival probabilities using the parameter $c_{1}$ to the model $\frac{1}{2}+B_{0} \exp \left(-c_{1} n_{k}\right)$, where $B_{0}$ is determined by the fixed $Z$-dephasing noise power and $n_{k}$ the number of $R_{x}(\pi)$ pulses in the $k$ th FTTPS. (b) Ideal $R_{x}(\pi)$ gates are replaced by over-rotated gates $R_{x}(\pi+\vartheta)$ and $R_{x}(\pi / 2)$ gates with $R_{x}((\pi+\vartheta) / 2)$. The dashed line shows least-squares fits using the parameter $c_{2}$ to the model $\frac{1}{2}+B_{0} \exp \left(-c_{2} n_{k}^{2}\right)$. The solid lines show fits using an additional parameter $\beta$ for a model $\frac{1}{2}+B_{0} \exp \left(-c_{2} n_{k}^{\beta}\right)$ which improves the fits for the larger errors.

different standard deviations $\sigma$. As expected from the analytical expressions, the FTTPS conveys reductions in survival probability that increase with probe sequence number, i.e., number of pulses. In addition, comparing the FTTPS (top row) and RFTTPS (bottom row), we find that the robust sequences suppress coherent errors extremely well, while stochastic errors typically do not benefit from the phase alterations used in RFTTPSs.

We corroborate our analytical results with a series of SchWARMA-driven numerical experiments using FTTPS under different $R_{x}(\pi)$-gate error models. In the first set of experiments, we consider fixed power white noise-driven $Z$-dephasing errors and followed each $R_{x}(\theta)$ gate with a random $R_{x}(\vartheta)$ gate, with $\vartheta$ drawn independently from a zero-mean normal distribution with standard deviation $\sigma$ (we note that this is an example of a gate-dependent, multiaxis SchWARMA model). Figure 13(a) shows the resulting survival probabilities for various $\sigma$, along with fits to an exponential decay model proportional to $n_{k}$, the number of $R_{x}(\pi)$ gates in a given sequence. These exponential decays fit the resulting simulated survival probabilities quite well, and we note that for modest $\sigma$, the decay appears quite linear.

The next error model we considered was a fixed, coherent over-rotation error on the $R_{x}(\theta)$ gates, subject to the same $Z$-dephasing error as above; this models a long-running calibration error. The over-rotation was held constant for the full set of FTTPSs and all Monte Carlo trajectories. Specifically, we replaced ideal $R_{x}(\pi)$ gates with $R_{x}(\pi+\vartheta)$ and $R_{x}(\pi / 2)$ gates with $R_{x}((\pi+\vartheta) / 2)$ for several values of $\vartheta$, see Fig. 13(b). Again, we performed least-squares fits to the survival probabilities, but instead of an exponential decay pro- portional to $n_{k}$, we intuitively expected a decay proportional to $n_{k}^{2}$. We found that this model fits quite well for modest $\vartheta$, but was not a great fit for $\vartheta=0.01 \mathrm{rad}$. To see if we could produce a better fit, we added an additional regression parameter $\beta$ and performed fits of exponential decay proportional to $n_{k}^{\beta}$. We saw that this improved the mean squared error of the fit to $\vartheta=0.01$ rad by nearly an order of magnitude, produced a modest improvement in the $\vartheta=0.0075 \mathrm{rad}$ case, and negligible differences in the remaining.

In summary, these simulations further support the hypothesis that the high-frequency noise observed in the FTTPS-based spectrum reconstructions are likely due to presence of gate errors. Thus, when performing SchWARMA-driven predictions and fits, it is necessary to incorporate terms that account for effects which the results above indicate can be quite well modeled using only a few terms (i.e., $c_{1}, c_{2}$, and $\beta$ ). Furthermore, since the decays we observed in the experimental data are not at the extremes of what we explored in simulation, the inclusion of the $\beta$ term is not needed. We also note that we found that the inclusion of the $c_{1}$ term can improve prediction accuracy even when the coherent errors are canceled out using alternating directions in the $R_{x}(\pi)$ pulses.

\section{Experimental implementation}

We perform a comparison between FTTPSs and RFTTPSs on the IBM Armonk processor under the condition of native and injected noise. FTTPSs are constructed from $U$ gates of duration $t_{G} \approx 248 \mathrm{~ns}$, while RFTTPS are composed of true, precalibrated $\pi$ pulses of duration $t_{G} \approx 142 \mathrm{~ns}$. The Armonk processor maintains a library of precalibrated pulses that 

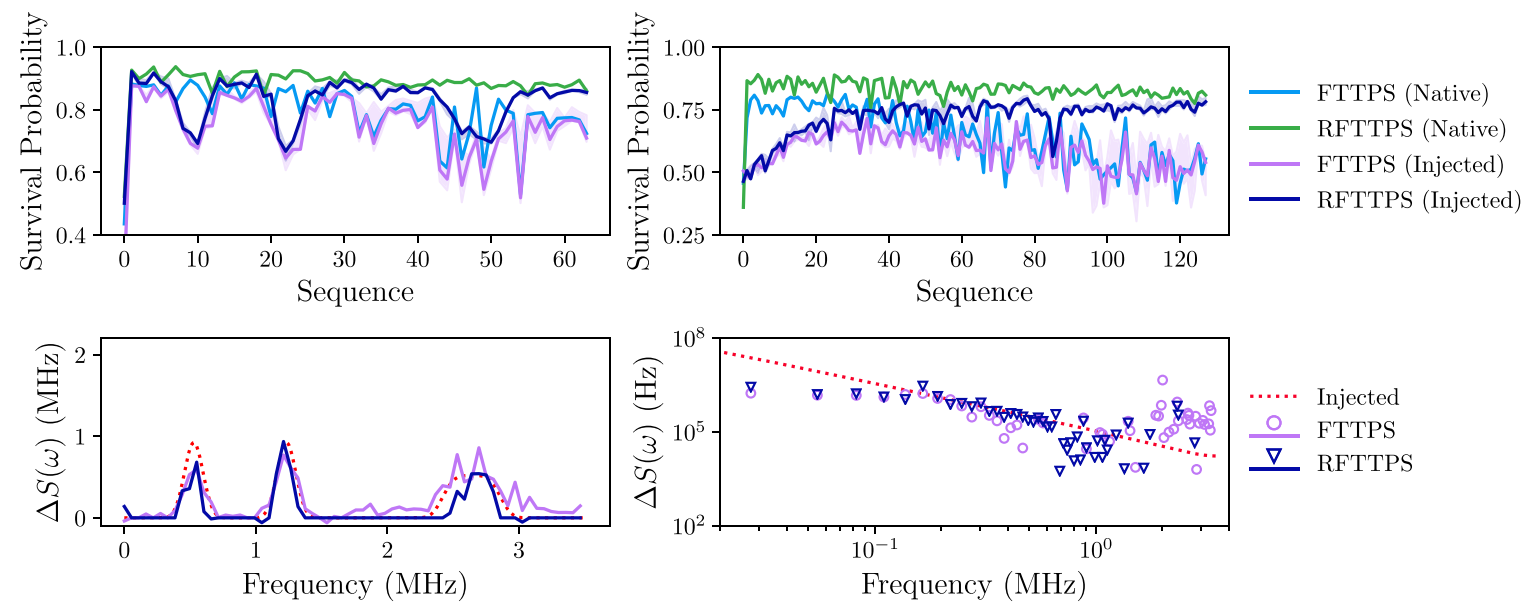

FIG. 14. Comparison of QNS protocols in the presence of native and injected $1 / f$ noise. Left: Survival probability as a function of probe sequence index for FTTPSs and RFTTPSs for both native and injected noise. Right: Spectrum estimates for FTTPS and RFFPS for injected noise. Experiments were performed on the IBMQE.

includes an $R_{x}(\pi)$ DRAG pulse that is consistently updated each calibration cycle. By altering the phase of the precalibrated DRAG pulse, we obtain the additional $R_{x}(-\pi)$ used in the RFTTPSs. In Fig. 14, the top-left panel displays the survival probabilities for both sets of probe sequences in the presence of native and injected triple bandpass noise bootstrapped over five calibration cycles. The first two bandpass signals possess the same bandwidth of $\Delta \omega=0.18 \mathrm{MHz}$, while the third, high-frequency bandpass signal has a bandwidth of $\Delta \omega=0.54 \mathrm{MHz}$. The center frequencies for the three bandpass signals are $\omega_{0}=0.53,1.23,2.64 \mathrm{MHz}$. The top-right panel of Fig. 14 shows a similar comparison for injected $1 / f$ noise. Native noise survival probabilities display a clear distinction between FTTPSs and RFTTPSs, most notably at large sequence index (i.e., high frequency) where pulse errors are more evident for FTTPSs than RFTTPSs.

By injecting noise and comparing estimated spectra to the expected injected noise spectra, we determine which protocol supplies the more accurate spectral estimates. The bottom panels of Fig. 14 show the resulting estimated injected spectra for triple bandpass (bottom left) and $1 / f$ (bottom right) noise. FTTPS spectral estimations exhibit deviations from the ideal spectrum at high frequency despite correction for the native spectrum. Specifically, these deviations can be ob- served in the bottom panels of Fig. 14 at frequencies greater than 1.5 MHz. Divergences from the ideal spectrum are less substantial for RFTTPSs, indicating that this particular set of probe sequences yields more accurate spectral estimates. Together, these comparisons convey the potential benefit of utilizing true $\pi$ pulses and phase adjustments to improve QNS estimation.

The comparison performed above and in the main text indicate that survival probabilities are correlated with the number of gates in a given FTTPS. Noting that this was inconsistent with the established literature on the dephasing spectra of superconducting qubits, we conjectured that this was primarily due to errors on the $R_{x}(\pi)$ gates. The comparison between nonrobust and robust variations of FTTPSs supports the claim that pulse errors indeed contribute to spurious features in the dephasing noise spectrum. However, while the RFTTPSs provide insight into ways of suppressing such features, they do not elucidate details about the noise characteristics of the pulse error. Based on our analytical and numerical analyses, we conjecture that the pulse errors are likely characterized by a correlation time that is more consistent with slow drift rather than rapidly fluctuating, uncorrelated errors. Estimating this correlation time is left for future studies of multiaxis noise spectroscopy protocols.
[1] Y. Romach, C. Müller, T. Unden, L. J. Rogers, T. Isoda, K. M. Itoh, M. Markham, A. Stacey, J. Meijer, S. Pezzagna, B. Naydenov, L. P. McGuinness, N. Bar-Gill, and F. Jelezko, Spectroscopy of Surface-Induced Noise Using Shallow Spins in Diamond, Phys. Rev. Lett. 114, 017601 (2015).

[2] V. M. Frey, S. Mavadia, L. M. Norris, W. de Ferranti, D. Lucarelli, L. Viola, and M. J. Biercuk, Application of optimal band-limited control protocols to quantum noise sensing, Nat. Commun. 8, 2189 (2017).

[3] K. W. Chan, W. Huang, C. H. Yang, J. C. C. Hwang, B. Hensen, T. Tanttu, F. E. Hudson, K. M. Itoh, A. Laucht, A. Morello, and A. S. Dzurak, Assessment of a Silicon Quantum Dot Spin
Qubit Environment Via Noise Spectroscopy, Phys. Rev. Appl. 10, 044017 (2018).

[4] Y. Sung, F. Beaudoin, L. M. Norris, F. Yan, D. K. Kim, J. Y. Qiu, W. von Lüpke, J. L. Yoder, T. P. Orlando, S. Gustavsson, L. Viola, and W. D. Oliver, Non-gaussian noise spectroscopy with a superconducting qubit sensor, Nat. Commun. 10, 3715 (2019).

[5] U. von Lüpke, F. Beaudoin, L. M. Norris, Y. Sung, R. Winik, J. Y. Qiu, M. Kjaergaard, D. Kim, J. Yoder, S. Gustavsson, L. Viola, and W. D. Oliver, Two-qubit spectroscopy of spatiotemporally correlated quantum noise in superconducting qubits, PRX Quantum 1, 010305 (2020). 
[6] R. Blume-Kohout, J. K. Gamble, E. Nielsen, K. Rudinger, J. Mizrahi, K. Fortier, and P. Maunz, Demonstration of qubit operations below a rigorous fault tolerance threshold with gate set tomography, Nat. Commun. 8, 14485 (2017).

[7] E. Knill, D. Leibfried, R. Reichle, J. Britton, R. B. Blakestad, J. D. Jost, C. Langer, R. Ozeri, S. Seidelin, and D. J. Wineland, Randomized benchmarking of quantum gates, Phys. Rev. A 77, 012307 (2008).

[8] E. Magesan, J. M. Gambetta, and J. Emerson, Scalable and Robust Randomized Benchmarking of Quantum Processes, Phys. Rev. Lett. 106, 180504 (2011).

[9] D. Lidar, Review of decoherence free subspaces, noiseless subsystems, and dynamical decoupling, Adv. Chem. Phys. 154, 295 (2014).

[10] L. Viola and S. Lloyd, Dynamical suppression of decoherence in two-state quantum systems, Phys. Rev. A 58, 2733 (1998).

[11] L. Viola, E. Knill, and S. Lloyd, Dynamical Decoupling of Open Quantum Systems, Phys. Rev. Lett. 82, 2417 (1999).

[12] P. Zanardi, Symmetrizing evolutions, Phys. Lett. A 258, 77 (1999).

[13] L. Viola, Introduction to quantum dynamical decoupling, in Quantum Error Correction, edited by D. A. Lidar and T. A. Brun (Cambridge University Press, Cambridge, UK, 2013), pp. 105-125.

[14] P. W. Shor, Scheme for reducing decoherence in quantum computer memory, Phys. Rev. A 52, R2493 (1995).

[15] A. M. Steane, Error Correcting Codes in Quantum Theory, Phys. Rev. Lett. 77, 793 (1996).

[16] P. Aliferis, D. Gottesman, and J. Preskill, Quantum accuracy threshold for concatenated distance-3 codes, Quantum Inf. Comput. 6, 97 (2006).

[17] B. M. Terhal, Quantum error correction for quantum memories, Rev. Mod. Phys. 87, 307 (2015).

[18] M. A. Nielsen and I. L. Chuang, Quantum Computation and Quantum Information: 10th Anniversary Edition, 10th ed. (Cambridge University Press, Cambridge, UK, 2011).

[19] I. L. Chuang, R. Laflamme, P. W. Shor, and W. H. Zurek, Quantum computers, factoring, and decoherence, Science 270, 1633 (1995).

[20] E. Knill, R. Laflamme, and W. H. Zurek, Resilient quantum computation: error models and thresholds, Proc. R. Soc. London 454, 365 (1998).

[21] E. Knill, R. Laflamme, and W. H. Zurek, Resilient quantum computation, Science 279, 342 (1998).

[22] J. I. Colless, V. V. Ramasesh, D. Dahlen, M. S. Blok, M. E. Kimchi-Schwartz, J. R. McClean, J. Carter, W. A. de Jong, and I. Siddiqi, Computation of Molecular Spectra on a Quantum Processor with an Error-Resilient Algorithm, Phys. Rev. X 8, 011021 (2018).

[23] J. R. McClean, M. E. Kimchi-Schwartz, J. Carter, and W. A. de Jong, Hybrid quantum-classical hierarchy for mitigation of decoherence and determination of excited states, Phys. Rev. A 95, 042308 (2017).

[24] S. Mavadia, C. L. Edmunds, C. Hempel, H. Ball, F. Roy, T. M. Stace, and M. J. Biercuk, Experimental quantum verification in the presence of temporally correlated noise, NPJ Quantun Inf. 4, 7 (2018).

[25] A. Soare, H. Ball, D. Hayes, X. Zhen, M. C. Jarratt, J. Sastrawan, H. Uys, and M. J. Biercuk, Experimental bath engi- neering for quantitative studies of quantum control, Phys. Rev. A 89, 042329 (2014).

[26] K. Schultz, G. Quiroz, P. Titum, and B. D. Clader, Schwarma: A model-based approach for time-correlated noise in quantum circuits, Phys. Rev. Res. 3, 033229 (2021).

[27] IBM Quantum Experience, https://quantum-computing.ibm. com.

[28] G. A. Álvarez and D. Suter, Measuring the Spectrum of Colored Noise by Dynamical Decoupling, Phys. Rev. Lett. 107, 230501 (2011).

[29] H. Ball, W. Oliver, and M. Biercuk, The role of master clock stability in quantum information processing, npj Quantum Inf 2, 16033 (2016).

[30] S. H. Holan, R. Lund, and G. Davis, The ARMA alphabet soup: A tour of ARMA model variants, Stat. Surv. 4, 232 (2010).

[31] S. Plaszczynski, Generating long streams of $1 / \mathrm{f} \alpha$ noise, Fluct. Noise Lett. 7, R1 (2007).

[32] D. C. McKay, C. J. Wood, S. Sheldon, J. M. Chow, and J. M. Gambetta, Efficient $z$ gates for quantum computing, Phys. Rev. A 96, 022330 (2017).

[33] Quantum computation center opens, https://www.ibm.com/ blogs/research/2019/09/quantum-computation-center/, accessed Aug. 7, 2021.

[34] J. Meeson, A. Ya. Tzalenchuk, and T. Lindström, Evidence for interacting two-level systems from the $1 / \mathrm{f}$ noise of a superconducting resonator, Nat. Commun. 5, 4119 (2014).

[35] J. J. Burnett, A. Bengtsson, M. Scigliuzzo, D. Niepce, M. Kudra, P. Delsing, and J. Bylander, Decoherence benchmarking of superconducting qubits, npj Quantum Inf. 5, 54 (2019).

[36] C. Müller, J. Lisenfeld, A. Shnirman, and S. Poletto, NonGaussian noise spectroscopy with a superconducting qubit sensor, Phys. Rev. B 92, 035442 (2015).

[37] J. Bylander, S. Gustavsson, F. Yan, F. Yoshihara, K. Harrabi, G. Fitch, D. G. Cory, Y. Nakamura, J.-S. Tsia, and W. D. Oliver, Noise spectroscopy through dynamical decoupling with a superconducting flux qubit, Nat. Phys. 7, 565 (2019).

[38] F. Yan, S. Gustavsson, J. Bylander, X. Jin, F. Yoshihara, D. G. Cory, Y. Nakamura, T. P. Orlando, and W. D. Oliver, Rotationg-frame relaxation as a noise spectrum analyser of a superconducting qubit undergoing driven evolution, Nat. Commun. 4, 2337 (2013).

[39] J. Basset, A. Stockklauser, D.-D. Jarausch, T. Frey, C. Reichl, W. Wegscheider, A. Wallraff, K. Ensslin, and I. T, Evaluating charge noise acting on semiconductor quantum dots in the circuit quantum electrodynamics architecture, Appl. Phys. Lett. 105, 063105 (2014).

[40] T. Struck, A. Hollmann, F. Schauer, O. Fedorets, A Schmidbauer, K. Sawano, H. Riemann, N. V. Abrosimov, L. Cywiński, D. Bougeard, and L. R. Schreiber, Low-frequency spin qubit energy splitting noise in highly purified ${ }^{28} \mathrm{Si} / \mathrm{SiGe}$, npj Quantum Inf. 6, 40 (2020).

[41] F. Yan, S. Gustavsson, A. Kamal, J. Birenbaum, A. P. Sears, D. Hover, T. J. Gudmundsen, D. Rosenberg, G. Samach, S. Weber et al., The flux qubit revisited to enhance coherence and reproducibility, Nat. Commun. 7, 12964 (2016).

[42] T. A. Alexander, N. Kanazawa, D. J. Egger, L. Capelluto, C. J. Wood, A. Javadi-Abhari, and D. McKay, Qiskit-pulse: 
Programming quantum computers through the cloud with pulses, Quantum Sci. Technol. 5, 044006 (2020).

[43] R. L. Vold and H. E. Simon, Errors in measurements of transverse relaxation rates, J. Magn. Reson. (1969) 11, 283 (1973).

[44] M. H. Levitt and R. Freeman, Composite pulse decoupling, J. Magn. Reson. (1969) 43, 502 (1981).

[45] S. T. Smith, Bounded-strength dynamical control of a qubit based on Eulerian cycles, Ph.D. thesis, Dartmouth College, 2007.

[46] G. Quiroz and D. A. Lidar, Optimized dynamical decoupling via genetic algorithms, Phys. Rev. A 88, 052306 (2013).

[47] J. J. Wallman and J. Emerson, Noise tailoring for scalable quantum computation via randomized compiling, Phys. Rev. A 94, 052325 (2016).
[48] M. Ware, G. Ribeill, D. Ristè, C. A. Ryan, B. Johnson, and M. P. da Silva, Experimental demonstration of Pauli-frame randomization on a superconducting qubit, Phys. Rev. A 103, 042604 (2021).

[49] P. Titum, K. M. Schultz, A. Seif, G. D. Quiroz, and B. Clader, Optimal control for quantum detectors, npj Quantum Inf. 7, 53 (2021).

[50] V. Frey, L. M. Norris, L. Viola, and M. J. Biercuk, Optimally Band-Limited Controls for Quantum Multi-Axis Spectral Estimation, Phys. Rev. Appl. 14, 024021 (2020).

[51] L. M. Norris, D. Lucarelli, V. M. Frey, S. Mavadia, M. J. Biercuk, and L. Viola, Optimally band-limited spectroscopy of control noise using a qubit sensor, Phys. Rev. A 98, 032315 (2018). 